\title{
FOURIER COEFFICIENTS OF GENERALIZED EISENSTEIN SERIES OF DEGREE TWO II
}

\author{
By SHIN-ICHIRo Mizumoto
}

\section{$\S 1$. Statement of Results.}

This is a continuation of Part I ([13]). We treat a generalization of the previous result and investigate a non-vanishing property of Fourier coefficients. We follow the notation of Part I throughout this paper.

Let $f \in M_{k}\left(\Gamma_{1}\right)$ be a normalized elliptic eigen modular form of weight $k$, and let $[f] \in M_{k}\left(\Gamma_{2}\right)$ be the generalized Eisenstein series of degree two attached to $f$ in the sense of Langlands [8] and Klingen [4] (see (1.4) of Part I). Then [f] is an eigen modular form of weight $k$ satisfying $\Phi([f])=f$ where $\Phi$ is the Siegel operator (Proposition in $\S 2$ of Part I); conversely, by Kurokawa [5], [f] is characterized by these properties. Let

$$
[f](Z)=\sum_{T \geq 0} a(T,[f]) \mathrm{e}(\operatorname{tr}(T Z))
$$

be the Fourier expansion of $[f]$, where $\mathrm{e}(z)=\exp (2 \pi i z)$, tr denotes the trace of matrices, and $T$ runs over all symmetric positive semi-definite semi-integral matrices of size 2 . We investigate these $a(T,[f])$.

Let

$$
f(z)=\sum_{n=0}^{\infty} a(n) \mathrm{e}(n z)
$$

be the Fourier expansion of $f$, hence $T(n) f=a(n) f$ for each $n \geqq 1$. For $|T|=0$ we have $a(T,[f])=a(n)$ if $T$ is unimodularly equivalent to $\left(\begin{array}{ll}n & 0 \\ 0 & 0\end{array}\right)$, since $\Phi([f])$ $=f$. For $T>0$, from $T(p)[f]=\left(1+p^{k-2}\right) a(p)[f]$ ( $p$ a prime number) we have:

$$
\begin{aligned}
a(p T,[f])= & \left(1+p^{k-2}\right) a(p) a(T,[f])-p^{2 k-3} a\left(p^{-1} T,[f]\right) \\
& -p^{k-2} a\left(p^{-1} T\left[\begin{array}{ll}
0 & p \\
1 & 0
\end{array}\right],[f]\right)-p^{k-2} \sum_{j=0}^{p-1} a\left(p^{-1} T\left[\begin{array}{ll}
1 & 0 \\
j & p
\end{array}\right],[f]\right)
\end{aligned}
$$

as in MaaB $[11]$. Here we understand that $a(*,[f])=0$ unless $*$ is semi-integral. The relation (1.3) enables us to write each $a(T,[f])$ as an explicit $\boldsymbol{Z}(f)$-linear combination of $a(T,[f])$ for primitive $T>0$, where $\boldsymbol{Z}(f)$ is the integer ring of the totally real number field $\boldsymbol{Q}(f)=\boldsymbol{Q}(a(n) \mid n \geqq 1)$ and we say that $T=\left(\begin{array}{cc}t_{1} & t / 2 \\ t / 2 & t_{2}\end{array}\right)$

Received March 25, 1983 
is primitive if g.c.d. $\left(t_{1}, t_{2}, t\right)=1$. Thus we may limit our attention to the case where $T>0$ is primitive. We put $|2 T|=\Delta(T) \mathfrak{f}^{2}$ with a positive integer $\mathfrak{f}$, where $-\Delta(T)$ is the discriminant of the quadratic field $\boldsymbol{Q}(\sqrt{-|2 T|})$. We denote by $\chi=\left(\frac{-\Delta(T)}{*}\right)$ the Dirichlet character associated with $\boldsymbol{Q}(\sqrt{-|2 T|})$.

Now we state our main result.

THEOREM 1. Let $f$ be a normalized eigen modular form of weight $k$ with respect to $\Gamma_{1}=\mathrm{SL}(2, \boldsymbol{Z})$ with a Fourier expansion (1.2). Suppose $T>0$ is primitive. Then we have:

$$
\begin{aligned}
a(T,[f])= & (-1)^{k / 2} \frac{(k-1) !}{(2 k-2) !}(2 \pi)^{k-1} \Delta(T)^{k-(3 / 2)} \frac{L(k-1, \chi)}{L_{2}(2 k-2, f)} \\
& \cdot \sum_{\substack{s>f \\
s>0}} M\left(\mathfrak{f} s^{-1}\right) \sum_{\substack{t>s \\
t>0}} \mu(t) D\left(k-1, f, \vartheta_{T}^{(s / t)}\right) .
\end{aligned}
$$

Here

$$
L_{2}(s, f)=\prod_{p}\left(1-\alpha_{p}^{2} p^{-s}\right)^{-1}\left(1-\alpha_{p} \beta_{p} p^{-s}\right)^{-1}\left(1-\beta_{p}^{2} p^{-s}\right)^{-1}
$$

is the "second" $L$-function attached to $f, \alpha_{p}$ and $\beta_{p}$ being complex numbers such that $\alpha_{p}+\beta_{p}=a(p)$ and $\alpha_{p} \beta_{p}=p^{k-1}$,

$$
\begin{gathered}
\vartheta_{T}(z)=\sum_{(m, n) \in Z^{2}} \mathrm{e}\left(z\left(t_{1} m^{2}+t m n+t_{2} n^{2}\right)\right) \quad \text { for } \quad T=\left(\begin{array}{cc}
t_{1} & t / 2 \\
t / 2 & t_{2}
\end{array}\right), \\
D\left(s, f, \vartheta_{T}^{(v)}\right)=\sum_{n=1}^{\infty} a(n) b_{T}\left(v^{2} n\right) n^{-s} \quad \text { if we write } \quad \vartheta_{T}(z)=\sum_{n=0}^{\infty} b_{T}(n) \mathrm{e}(n z),
\end{gathered}
$$

$L(s, \chi)$ is the Dirichlet $L$-function, $\mu$ is the Möbius function, and

$$
M(a)=\sum_{\substack{d \backslash a \\ d>0}} \mu(d) \chi(d) d^{k-2} \sigma_{2 k-3}\left(a d^{-1}\right)
$$

where $\sigma_{s}(d)=\sum_{\substack{g|d| \\ g>0}} g^{s}$. We understand that each $L$-function is analytically continued to the whole $\stackrel{g}{s}$-plane.

We note that a direct computation shows that (1.4) is equivalent to:

$$
\begin{aligned}
a(T,[f])= & (-1)^{k / 2} \frac{(k-1) !}{(2 k-2) !}(2 \pi)^{k-1} \Delta(T)^{k-(3 / 2)} \frac{L(k-1, \chi)}{L_{2}(2 k-2, f)} \\
& \cdot \sum_{\substack{v \mid \uparrow \\
v>0}} D\left(k-1, f, \varphi_{T, v}\right) .
\end{aligned}
$$

where

$$
\varphi_{T, v}=\left(\mathfrak{f} v^{-1}\right)^{2 k-3} \prod_{\substack{p \mid \mathfrak{f} v^{-1} \\ p: \operatorname{pr} 1 \mathrm{me}}}\left(1-\chi(p) p^{1-k}\right) \vartheta_{T}^{(v)} .
$$

In case $\Phi f \neq 0$, i. e. $f(z)=G_{k}(z)=-\frac{B_{k}}{2 k}+\sum_{n=1}^{\infty} \sigma_{k-1}(n) \mathrm{e}(n z) \quad(k$ is an even integer $\geqq 4$ ), then, as we shall see in $\S 3$, (1.4) may also be written : 


$$
a\left(T,\left[G_{k}\right]\right)=(-1)^{k / 2} \frac{(k-1) !}{(2 k-2) !} 2(2 \pi)^{k-1} \Delta(T)^{k-(3 / 2)} \frac{L(k-1, \chi)}{\zeta(2 k-2)} M(\mathfrak{f})
$$

which coincides with the formula of Maaß $[11]$ for $a\left(T,\left[G_{k}\right]\right)$.

Here we note that all $a(T,[f])$ have unified expression for both cases $\Phi f=0$ and $\Phi f \neq 0$, by Theorem 1 and by the relation (1.3). This suggests that the higher degree cases are in similar situations.

Exactly as in Part I, we have the following algebraicity result from Theorem 1 and (1.3) combined with the algebraicity concerning $D\left(k-1, f, \vartheta_{T}^{(v)}\right)$ and $L_{2}(2 k-2, f)$ shown by Shimura [15], Sturm [17], and Zagier [18]:

THEOREM 2. Let $f$ be as in Theorem 1. Then, for all $T \geqq 0$ we have:

(1) $a(T,[f])^{\sigma}=a\left(T,\left[f^{\sigma}\right]\right)$ for all $\sigma \in \operatorname{Aut}(\boldsymbol{C})$.

(2) $a(T,[f])$ belong to $\boldsymbol{Q}(f)$.

Here $\boldsymbol{Q}(f)=\boldsymbol{Q}(a(n) \mid n \geqq 1)$. Theorem 2 is a special case of the result of Kurokawa [5].

In $\S 4$ we treat a non-vanishing problem suggested by Professor Maaß.

THEOREM 3. Let $f$ be as in Theorem 1. Suppose that $T \geqq 0$ (as a quadratic form) represents 1 over $\boldsymbol{Z}$. Then : $a(T,[f]) \neq 0$.

The author would like to thank Professor H. Maaß and Professor N. Kurokawa for suggestions and encouragements.

Notes. (1) A generalization to the Hilbert-Siegel modular case is treated in [14]. (2) Theorem 1 for the case $\Phi f=0$ is also obtained by S. Böcherer "Über das Verhalten der Fourierentwicklung bei Liftung von Modulformen" (thesis, AlbertLudwigs-Universität, Freiburg, 1981) using a different method ; Professor Böcherer communicated his result to the author in October 1981.

\section{Notation.}

$1^{\circ}$. The letter $p$ always denotes a prime number, and $\prod_{p}$ means the product taken over all prime numbers. For an integer $a$, the fact " $p^{t} \mid a$ and $p^{t+1} \nmid a$ " is denoted by $p^{t} \| a$.

$2^{\circ}$. For a set $X$, its cardinality is denoted by $\# X$.

$3^{\circ}$. For a real number $x,[x]$ is the maximal integer $\leqq x$.

$4^{\circ}$. We understand that summation (resp. product) over the empty set is equal to 0 (resp. 1).

$5^{\circ}$. If $A$ is a ring with 1 , then $A^{\times}$denotes the group of units.

\section{$\S 2$. Proof of Theorem 1: The Case $\Phi f=0$.}

Throughout this section we suppose that $f$ is a normalized eigen cusp form with the Fourier expansion (1.2) with $a(0)=0$. By (3.5) in Part I, for each $T>0$ 
we have:

where

$$
\begin{aligned}
& a(T,[f])=\xi|T|^{k-(3 / 2)} \sum_{(r, s) \in \mathscr{P}} \sum_{\substack{2 \\
d^{2} Q(r, s) \\
d>0}}\left\{a\left(Q(r, s) d^{-2}\right) Q(r, s)^{1-k}\right. \\
& \left.\cdot \sum_{g=1}^{\infty} g^{-k} \sum_{\sigma \bmod g} \sum_{g} \mathrm{e}\left(R_{r, s, \sigma} \rho / g d\right) K_{g}\left(Q(r, s) d^{-2} \rho^{2}, Q\left(u_{0}+\sigma r, v_{0}+\sigma s\right)\right)\right\}
\end{aligned}
$$

$$
\begin{gathered}
\mathscr{P}=\left\{(x, y) \in Z^{2} \mid \begin{array}{l}
x \text { and } y \text { are relatively prime; } \\
x>0 \text { or }(x, y)=(0,1) .
\end{array}\right\} ; \\
\left(\begin{array}{cc}
Q(r, s) & R_{r, s, \sigma} / 2 \\
R_{r, s, \sigma} / 2 & Q\left(u_{0}+\sigma r, v_{0}+\sigma s\right)
\end{array}\right)=T\left[\begin{array}{ll}
r & u_{0}+\sigma r \\
s & v_{0}+\sigma s
\end{array}\right]
\end{gathered}
$$

with integers $u_{0}, v_{0}$ satisfying $r v_{0}-s u_{0}=1$; for $a, b \in \boldsymbol{Z}$ we put

$$
K_{g}(a, b)=\sum_{\substack{x \bmod g \\(x, g)=1}} \mathrm{e}\left(\left(a x+b x^{-1}\right) / g\right),
$$

$\left(x^{-1}\right.$ denoting an integer such that $\left.x x^{-1} \equiv 1(\bmod g)\right)$; and

$$
\xi=(-1)^{k / 2} 2^{k-1} \pi^{k-(1 / 2)} \Gamma(k-(1 / 2))^{-1} .
$$

We investigate the exponential sums involving $K_{g}$. For a while we fix $(r, s) \in \mathscr{L}$ and put

$$
T\left[\begin{array}{ll}
r & u_{0} \\
s & v_{0}
\end{array}\right]=\left(\begin{array}{cc}
l & m / 2 \\
m / 2 & n
\end{array}\right)
$$

so $T\left[\begin{array}{cc}r & u_{0}+\sigma r \\ s & v_{0}+\sigma s\end{array}\right]=\left(\begin{array}{cc}l & m / 2 \\ m / 2 & n\end{array}\right)\left[\begin{array}{ll}1 & \sigma \\ 0 & 1\end{array}\right]$. Note that: $Q(r, s)=l, R_{r, s, \sigma}=2 l \sigma+m$, and $Q\left(u_{0}+\sigma r, v_{0}+\sigma s\right)=l \sigma^{2}+m \sigma+n$. The sum in question is :

$$
\begin{aligned}
& \Lambda(d, g)=\Lambda(l, m, n ; d, g) \\
& =\sum_{\sigma \bmod g} \sum_{\substack{\bmod d \\
(\rho, d)=1}} \mathrm{e}((2 l \sigma+m) \rho / g d) K_{g}\left(d^{-2} l \rho^{2}, l \sigma^{2}+m \sigma+n\right),
\end{aligned}
$$

where $d^{2} \mid l$.

LEMMA 1. $\Lambda(d, g)$ is simultaneously multiplicative in the following sense: if $d=\prod_{i} p_{i}^{\alpha}, g=\prod_{i} p_{i}^{\beta_{i}}$ with distinct primes $p_{i}$ and non-negative integers $\alpha_{i}, \beta_{i}$, then $\Lambda(d, g)=\prod_{i} \Lambda\left(p_{i}^{\alpha}, p_{i}^{\beta} i\right)$.

Proof. By (2.2) we have

$$
K_{g}(a, b)=\varphi(g d)^{-1} \varphi(g) \sum_{\substack{x \bmod , g d \\(x, g d)=1}} \mathrm{e}\left(\left(a x+b x^{-1}\right) / g\right)
$$

for each integer $d>0$, where $\varphi$ is the Euler's function. Thus 


$$
\begin{gathered}
\Lambda(d, g)=\varphi(g d)^{-1} \varphi(g) \sum_{\sigma \bmod g} \sum_{\substack{x \bmod g d \\
(x, g d)=1}} \sum_{\substack{\rho \bmod d \\
(\rho, d)=1}} \mathrm{e}\left((g d)^{-1}(2 l \sigma+m) \rho\right. \\
\left.+g^{-1}\left\{x^{-1} d^{-2} l \rho^{2}+x\left(\sigma^{2} l+\sigma m+n\right)\right\}\right) .
\end{gathered}
$$

Here we may replace $\rho$ by $\rho x$, since each $x$ satisfies $(x, d)=1$. Hence

$$
\begin{aligned}
& \Lambda(d, g)= \varphi(g d)^{-1} \varphi(g) \sum_{\sigma \bmod g} \sum_{\substack{x \bmod g d \\
(x, g d)=1}} \sum_{\substack{\rho \bmod d \\
(\rho, d)=1}} \\
& \cdot \mathrm{e}\left((g d)^{-1} x\left\{(2 l \sigma+m) \rho+d^{-1} l \rho^{2}+d\left(\sigma^{2} l+\sigma m+n\right)\right\}\right) \\
&= \varphi(g d)^{-1} \varphi(g) \sum_{\sigma \bmod g} \sum_{\substack{x \bmod g d \\
(x, g d)=1}} \sum_{\substack{\rho \bmod d \\
(\rho, d)=1}} \\
& \cdot \mathrm{e}\left((g d)^{-1} x\left\{d^{-1} l(\rho+d \sigma)^{2}+m(\rho+d \sigma)+d n\right\}\right) \\
&=\varphi(g d)^{-1} \varphi(g) \sum_{\substack{x \bmod g d \\
(x, g d)=1}} \sum_{\substack{\bmod g d \\
(\rho, d)=1}} \mathrm{e}\left((g d)^{-1} x\left(d^{-1} l \rho^{2}+m \rho+d n\right)\right) .
\end{aligned}
$$

Now suppose that $g=g_{1} g_{2}$ and $d=d_{1} d_{2}$ with positive integers $g_{1}, g_{2}, d_{1}, d_{2}$ such that $\left(g_{1} d_{1}, g_{2} d_{2}\right)=1$. Then, the elements of the set

$$
\{x \bmod g d \mid(x, g d)=1\} \quad(\text { resp. }\{\rho \bmod g d \mid(\rho, d)=1\})
$$

correspond bijectively to those of

$$
\prod_{j=1,2}\left\{x_{j} \bmod g_{j} d_{j} \mid\left(x_{j}, g_{j} d_{j}\right)=1\right\} \quad\left(\text { resp. } \prod_{j=1,2}\left\{\rho_{j} \bmod g_{j} d_{j} \mid\left(\rho_{j}, d_{j}\right)=1\right\}\right)
$$

through the relation $x=x_{1} g_{2} d_{2}+x_{2} g_{1} d_{1}$ (resp. $\rho=\rho_{1} g_{2} d_{2}+\rho_{2} g_{1} d_{1}$ ). Therefore, noting that $\left(d_{1} d_{2}\right)^{2} \mid l$, we have:

$$
\begin{aligned}
\Lambda(d, g)= & \varphi\left(g_{1}\right) \varphi\left(g_{1} d_{1}\right)^{-1} \sum_{\substack{x_{1} \bmod g_{1} d_{1} \\
\left(x_{1}, g_{1} d_{1}\right)=1}} \sum_{\substack{\rho_{1} \bmod g_{1} d_{1} \\
\left(\rho_{1}, d_{1}\right)=1}} \\
& \cdot \mathrm{e}\left(\left(g_{1} d_{1}\right)^{-1} d_{2} x_{1}\left\{d_{1}^{-1} l\left(\rho_{1} g_{2}\right)^{2}+m\left(\rho_{1} g_{2}\right)+d_{1} n\right\}\right) \\
& \cdot \varphi\left(g_{2}\right) \varphi\left(g_{2} d_{2}\right)^{-1} \sum_{\substack{x_{2} \\
\left(x_{2}, g_{2} g_{2}\right)=1}} \sum_{g_{2} d_{2} d_{2} \bmod g_{2} d_{2}} \\
& \cdot \mathrm{e}\left(\left(g_{2} d_{2}\right)^{-1} d_{1} x_{2}\left\{d_{2}^{-1} l\left(\rho_{2} g_{1}\right)^{2}+m\left(\rho_{2} g_{1}\right)+d_{2} n\right\}\right) \\
= & \Lambda\left(d_{1}, g_{1}\right) \Lambda\left(d_{2}, g_{2}\right)
\end{aligned}
$$

by (2.6), which shows that $\Lambda(d, g)$ is simultaneously multiplicative.

Remark 1. If $-|2 T|$ is a fundamental discriminant, we do not need Lemma 1 to compute the values of $\Lambda(d, g)$ (cf. Part I, Lemma 3 ).

Let $p$ be a prime number and $\gamma, \delta$ be non-negative integers such that $p^{2 \gamma} \mid l$. We calculate the value of $\Lambda\left(p^{\gamma}, p^{\delta}\right)=\Lambda\left(l, m, n ; p^{\gamma}, p^{\delta}\right)$. If $\delta=0$, then by (2.5), 


$$
\Lambda\left(p^{\gamma}, 1\right)=\sum_{\substack{\rho \bmod p \gamma \\
(\rho, p \gamma)=1}} \mathrm{e}\left(p^{-\gamma} m \rho\right)=\left\{\begin{array}{lll}
\varphi\left(p^{\gamma}\right) & \text { if } & p^{2 \gamma}|| 2 T \mid, \\
-p^{\gamma-1} & \text { if } & p^{2 \gamma-2} \||2 T|, \\
0 & \text { if } & p^{2 \gamma-2} \chi|2 T| .
\end{array}\right.
$$

If $\delta>0$, then by (2.6) we have:

$$
\begin{aligned}
\Lambda\left(p^{\gamma}, p^{\delta}\right) & =p^{-\gamma} \sum_{x} \sum_{\substack{\bmod p \gamma+\delta)=1 \\
(x, p)=1}} \sum_{\substack{\bmod p \gamma+\delta \\
(\rho)=1}} \mathrm{e}\left(p^{-\gamma-\delta} x\left(p^{-\gamma} l \rho^{2}+m \rho+p^{\gamma} n\right)\right) \\
& =p^{-\gamma} p^{\gamma+\delta}(A(p ; \gamma, \delta)-A(p ; \gamma, \delta-1)),
\end{aligned}
$$

where

$$
A(p ; \gamma, u)=\#\left\{\begin{array}{l|l}
\rho \bmod p^{\gamma+u} & \begin{array}{l}
p^{-\gamma} l \rho^{2}+m \rho+p^{\gamma} n \equiv 0\left(\bmod p^{\gamma+u}\right), \\
\left(\rho, p^{\gamma}\right)=1
\end{array}
\end{array}\right\} .
$$

Note that $A(p ; \gamma, u)=0$ if $p^{\gamma} \chi m$, since $p^{2 \gamma} \mid l$. Hence

$$
\Lambda\left(p^{\gamma}, p^{\delta}\right)=0 \quad \text { if } \delta>0, p^{2 \gamma} \times|2 T| .
$$

Next suppose $p^{2 \gamma}\left|l, p^{r}\right| m$. Then

where

$$
A(p ; \gamma, u)= \begin{cases}\varphi\left(p^{\gamma}\right) & \text { if } u=0 \\ p^{\gamma} B(p ; \gamma, u) & \text { if } u>0\end{cases}
$$

$$
B(p ; \gamma, u)=\#\left\{\begin{array}{ll}
\rho \bmod p^{u} & \begin{array}{l}
p^{-2 \gamma} l \rho^{2}+p^{-\gamma} m \rho+n \equiv 0\left(\bmod p^{u}\right) \\
\left(\rho, p^{\gamma}\right)=1
\end{array}
\end{array}\right\} .
$$

\begin{tabular}{|c|c|c|c|c|}
\hline & \multicolumn{2}{|c|}{$p:$ odd prime } & \multicolumn{2}{|c|}{$p=2$} \\
\hline \multirow{4}{*}{$p \nmid p^{-2 \gamma} l$} & \multirow{3}{*}{$1 \leqq u \leqq b-2 \gamma$} & \multirow{3}{*}{$p^{[u / 2]}$} & $1 \leqq u \leqq b-2 \gamma-2$ & $2^{[u / 2]}$ \\
\hline & & & $u=b-2 \gamma-1$ & $\delta(b / 2) 2^{(b / 2)-\gamma-1}$ \\
\hline & & & $u=b-2 \gamma$ & $\delta(b / 2) \chi(2)^{2} 2^{(b / 2)-\gamma}$ \\
\hline & $u \geqq b-2 \gamma+1$ & \multicolumn{3}{|c|}{$\delta(b / 2) \chi(p)(1+\chi(p)) p^{(b / 2)-\gamma}$} \\
\hline \multirow{2}{*}{$p \mid p^{-2 \gamma} l$} & $\gamma<b / 2$ & \multicolumn{3}{|c|}{0} \\
\hline & $\gamma=b / 2$ & \multicolumn{3}{|c|}{$\chi(p)(1+\chi(p)) / 2$} \\
\hline
\end{tabular}

Hence,

$$
\Lambda\left(p^{\gamma}, p^{\delta}\right)=\left\{\begin{array}{l}
p\left(p^{\gamma} B(p ; \gamma, 1)-\varphi\left(p^{\gamma}\right)\right) \quad \text { if } \delta=1, p^{2 \gamma}|| 2 T \mid \\
p^{\gamma+\delta}(B(p ; \gamma, \delta)-B(p ; \gamma, \delta-1)) \quad \text { if } \delta \geqq 2, p^{2 \gamma}|| 2 T \mid .
\end{array}\right.
$$

LEMMA 2. Let the notation be as above. Take the non-negative integer $b$ such that $p^{b} \||2 T|$. Suppose that $T>0$ is primitive and that $p^{2 \gamma}\left|l, p^{2 \gamma}\right||2 T|$. Then, $B(p ; \gamma, u)(u \geqq 1)$ takes the following values: 
where $\delta(x)=1$ or 0 according as $x$ is an integer or not.

Proof. First, observe the following:

$\left(1^{\circ}\right)$ By the above assumptions, $(l, m, n)=1$; if $\gamma>0$, then $p \nmid n$.

$\left(2^{\circ}\right)$ If $\gamma>0$, by $(2.10)$ we have

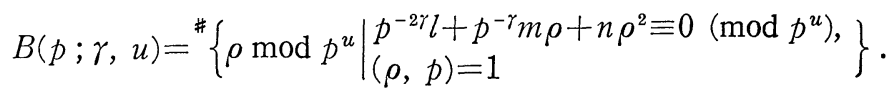

$\left(3^{\circ}\right)$ If $\gamma>0$ and $p \nmid p^{-2 \gamma} l$, then

$$
B(p ; \gamma, u)=\#\left\{\rho \bmod p^{u} \mid p^{-2 \gamma} l+p^{-\gamma} m \rho+n \rho^{2} \equiv 0\left(\bmod p^{u}\right)\right\} .
$$

$\left(4^{\circ}\right)$ Let $F(\rho)=p^{-2 \gamma} l+p^{-r} m \rho+n \rho^{2}$. Then its discriminant is equal to $-p^{-2 \gamma}|2 T|$. Namely, $4\left(p^{-2 r} l\right) n-\left(p^{-r} m\right)^{2}=p^{b-2 r} v$, where $|2 T|=p^{b} v$, $p \nmid v$.

$\left(5^{\circ}\right)$ By $\left(4^{\circ}\right)$ we have: $4 n F(\rho)-p^{-2 \gamma}|2 T|=\left(2 n \rho+p^{-r} m\right)^{2}$.

Now we examine each case in the table.

(i) $p \geqq 3, p \nmid p^{-2 r} l$.

If $\gamma>0$, then by $\left(1^{\circ}\right)\left(3^{\circ}\right)\left(5^{\circ}\right)$ we have

$$
B(p ; \gamma, u)=\#\left\{\rho \bmod p^{u} \mid\left(2 n \rho+p^{-\gamma} m\right)^{2} \equiv-p^{b-2 \gamma} v\left(\bmod p^{u}\right)\right\}
$$

and $p \nmid 2 n$. If $\gamma=0$, then by (2.10) we have

$$
\begin{aligned}
B(p ; \gamma, u) & =\#\left\{\rho \bmod p^{u} \mid l \rho^{2}+m \rho+n \equiv 0\left(\bmod p^{u}\right)\right\} \\
& =\#\left\{\rho \bmod p^{u} \mid(2 l \rho+m)^{2} \equiv-p^{b-2 \gamma} v\left(\bmod p^{u}\right)\right\},
\end{aligned}
$$

and $p \nmid 2 l$. Thus for each $\gamma \geqq 0, B(p ; \gamma, u)$ is equal to the number of the solutions of the congruence

$$
x^{2} \equiv-p^{b-2 \gamma} v\left(\bmod p^{u}\right),
$$

where $p \nmid v$.

(i-a) $u>b-2 \gamma$.

If $(2.12)$ is solvable, then it is necessary that $b \equiv 0(\bmod 2)$ and $\chi(p)=1$; here we note that $\left(\frac{-v}{p}\right)=\chi(p)$. Conversely, if these conditions are satisfied, then (2.12) is solvable and has $2 p^{(b-2 \gamma) / 2}$ solutions.

(i-b) $1 \leqq u \leqq b-2 \gamma$.

$$
B(p ; \gamma, u)=\#\left\{x \bmod p^{u} \mid x^{2} \equiv 0\left(\bmod p^{u}\right)\right\}=p^{[u / 2]} .
$$

(ii) $p \geqq 3, p \mid p^{-2 \gamma} l$.

Suppose first that $\gamma>0$, hence $p \nmid n$ by $\left(1^{\circ}\right)$. Then, from $\left(5^{\circ}\right)$ we have 


$$
\begin{aligned}
B(p ; \gamma, u) & =\#\left\{\begin{array}{ll}
\rho \bmod p^{u} & \begin{array}{l}
\left(2 n \rho+p^{-r} m\right)^{2} \equiv-p^{b-2 \gamma} v\left(\bmod p^{u}\right), \\
(\rho, p)=1
\end{array}
\end{array}\right\} \\
& =\left\{\left\{\begin{array}{ll}
x \bmod p^{u} & \begin{array}{l}
x^{2} \equiv-p^{b-2 \gamma} v\left(\bmod p^{u}\right), \\
x \neq p^{-r} m(\bmod p)
\end{array}
\end{array}\right\} .\right.
\end{aligned}
$$

Note that

$$
\left(p^{-r} m\right)^{2} \equiv-p^{b-2 \gamma} v(\bmod p)
$$

in this case. If $b>2 \gamma$, then (2.14) implies $p^{-\gamma} m \equiv 0(\bmod p)$, hence $B(p ; \gamma, u)=0$ by (2.13). If $b=2 \gamma$, then (2.14) implies that $p^{-r} m$ is one of the two solutions of $x^{2} \equiv-v(\bmod p)$, so we must have $\chi(p)=1$, and $B(p ; \gamma, u)=1$ by $(2.13)$.

Next suppose that $\gamma=0$. If moreover $b>0$, then by $\left(4^{\circ}\right)$ we have $p \mid m$, hence $p \nmid n$. Thus

$$
B(p ; 0, u)=\#\left\{\rho \bmod p^{u} \mid l \rho^{2}+m \rho+n \equiv 0\left(\bmod p^{u}\right)\right\}=0
$$

since $p|l, p| m, p \nmid n$. If $\gamma=b=0$, the proof of

$$
B(p ; 0, u)=\chi(p)(1+\chi(p)) / 2
$$

is similar to $\left(1^{\circ}\right)$ (iii) of the proof of Lemma 4 in Part I.

(iii) $p=2,2 \times 2^{-2 r} l$.

If $\gamma>0$, then by $\left(1^{\circ}\right)\left(3^{\circ}\right)\left(5^{\circ}\right)$,

$$
B(2 ; \gamma, u)=\#\left\{\rho \bmod 2^{u} \mid\left(2 n \rho+2^{-\gamma} m\right)^{2} \equiv-2^{b-2 \gamma} v\left(\bmod 2^{u+2}\right)\right\}
$$

and $2 \nmid n v$. If $\gamma=0$, then (2.10) implies

$$
B(2 ; \gamma, u)=\#\left\{\rho \bmod 2^{u} \mid(2 l \rho+m)^{2} \equiv-2^{b} v\left(\bmod 2^{u+2}\right)\right\}
$$

and $2 \nmid l v$. Hence for each $\gamma \geqq 0$ we have

$$
B(2 ; \gamma, u)=\#\left\{\rho \bmod 2^{u} \mid\left(2 \rho+2^{-\gamma} m\right)^{2} \equiv-2^{b-2 \gamma} v\left(\bmod 2^{u+2}\right)\right\} .
$$

Suppose $\gamma<b / 2$, hence $b-2 \gamma \geqq 2$. Then $2 \mid 2^{-\gamma} m$ and

$$
B(2 ; \gamma, u)=\#\left\{\rho \bmod 2^{u} \mid\left(\rho+2^{-\gamma-1} m\right)^{2} \equiv-2^{b-2 \gamma-2} v\left(\bmod 2^{u}\right)\right\} .
$$

Remaining argument is similar to (i).

Next suppose $\gamma=b / 2$. The congruence $x^{2} \equiv-v\left(\bmod 2^{u+2}\right)$ is solvable if and only if $\left(\frac{-v}{2}\right)=\chi(2)=1$, and if it is so, there exist four solutions. For each solution $x_{0}$ of this congruence, $\rho$ in the right hand side of (2.15) is determined by $2 \rho+2^{-\gamma} m \equiv x_{0}\left(\bmod 2^{u+1}\right)$; note that $2^{-\gamma} m \equiv x_{0}(\bmod 2)$. This yields two distinct values of $\rho \bmod 2^{u}$. Hence

$$
B(2 ; b / 2, u)=\delta(b / 2) \chi(2)(1+\chi(2)) .
$$


(iv) $p=2,2 \mid 2^{-2 \gamma}$.

Suppose $\gamma<b / 2$. Then $2 \mid 2^{-\gamma} m$, hence $2 \nmid n$. Thus by (2.10) we have

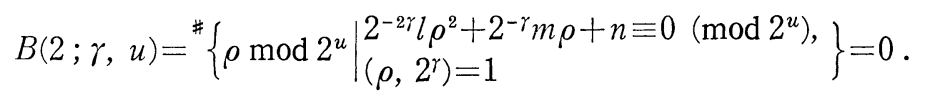

Next suppose that $\gamma=b / 2>0$. Then $2 \nmid n$ by $\left(1^{\circ}\right)$. As in (ii),

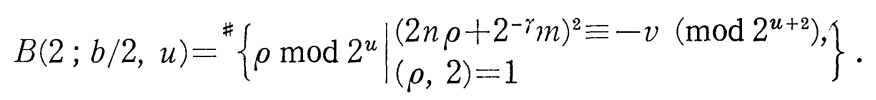

Then we proceed as in (iii), noting that $\rho$ is odd. Thus

$$
B(2 ; b / 2, u)=\delta(b / 2) \chi(2)(1+\chi(2)) / 2 .
$$

Finally suppose $b=2 \gamma=0$. In this case the proof is similar to $\left(1^{\circ}\right)$ (iv) of the proof of Lemma 4 in Part I.

Hereafter we suppose that $T>0$ is primitive. Then it follows from (2.7), (2.9), (2.11) and Lemma 2 that the values of $\Lambda(l, m, n ; d, g)$ depend only on $l, d, g$ whenever $(l, m, n)$ is defined by (2.4). So we write

and put

$$
\Lambda(l, m, n ; d, g)=\Lambda(l ; d, g)
$$

$$
S_{p}\left(l, p^{\Upsilon}\right)=\sum_{\delta=0}^{\infty} p^{-k \delta} \Lambda\left(l ; p^{\gamma}, p^{\delta}\right) .
$$

By Lemma 1,

$$
\sum_{g=1}^{\infty} g^{-k} \Lambda(l ; d, g)=\prod_{p} S_{p}\left(l,[d]_{p}\right),
$$

where $[d]_{p}$ denotes the $p$-part of $d$. Note that $S_{p}(l, 1) \neq 0$ since $\Lambda(l ; 1,1)=1$. Therefore (2.17) may also be written

$$
\sum_{g=1}^{\infty} g^{-k} \Lambda(l ; d, g)=\prod_{p} S_{p}(l, 1) \cdot \prod_{p \backslash d} S_{p}(l, 1)^{-1} S_{p}\left(l,[d]_{p}\right) .
$$

We compute the values of $S_{p}\left(l, p^{r}\right), p^{2 r} \mid l$. By (2.7) and (2.9),

$$
S_{p}\left(l, p^{\gamma}\right)= \begin{cases}0 & \text { if } r>0, p^{2 \gamma-2} \chi|2 T|, \\ -p^{\gamma-1} & \text { if } r>0, p^{2 \gamma-2} \||2 T| .\end{cases}
$$

So we next suppose that $p^{2 \gamma}|| 2 T \mid, \gamma>0$. Then by (2.7) and (2.11),

$$
\begin{aligned}
S_{p}\left(l, p^{\gamma}\right)= & \varphi\left(p^{\gamma}\right)+p^{1-k}\left(p^{\gamma} B(p ; \gamma, 1)-\varphi\left(p^{\gamma}\right)\right) \\
& +p^{\gamma} \sum_{\delta=2}^{\infty} p^{(1-k) \delta}(B(p ; \gamma, \delta)-B(p ; \gamma, \delta-1)),
\end{aligned}
$$

and by Lemma 2 we obtain the following values (after some computation). If $p \nmid p^{-2 \gamma} l$, then : 


$$
\begin{aligned}
S_{p}\left(l, p^{\gamma}\right)=- & \varepsilon(\gamma) p^{\gamma-1}\left(1-p^{1-k}\right)+p^{\gamma}\left(1-\chi(p) p^{1-k}\right)^{-1}\left(1-p^{2-2 k}\right) \\
& \cdot\left\{\left(1-\chi(p) p^{1-k}\right) \sum_{a=0}^{j-\gamma} p^{a(3-2 k)}+\chi(p)^{2} p^{(j-\gamma+1)(3-2 k)}\right\},
\end{aligned}
$$

where $\varepsilon(\gamma)=1$ or 0 according as $\gamma \geqq 1$ or $\gamma=0$, and $j=[(b-1) / 2]$ or $[(b-2) / 2]$ according as $p \geqq 3$ or $p=2$. If $p \mid p^{-2 \gamma} l$, then:

$$
S_{p}\left(l, p^{\gamma}\right)= \begin{cases}\left(1-p^{1-k}\right) \varphi\left(p^{\gamma}\right) & \text { for } \gamma<b / 2, \\ \left(1-p^{1-k}\right) \varphi\left(p^{\gamma}\right)+2^{-1} p^{\gamma+1-k} \chi(p)(1+\chi(p)) & \text { for } \gamma=b / 2 .\end{cases}
$$

In the above computation we use the following identity appearing also in MaaB [11]:

$$
\sum_{u=0}^{a} p^{(1-k) u} p^{[u / 2]}=\left(1+p^{1-k}\right) \sum_{n=0}^{[(a-1) / 2]} p^{u(3-2 k)}+\delta(a / 2) p^{a(3-2 k) / 2} .
$$

Now we return to (2.1) and derive a "product expression for $a(T,[f])$ ". As in Part I, let $H(-|2 T|)$ be the group of $\Gamma_{1}$-equivalence classes of primitive binary positive definite quadratic forms (which we identify with semi-integral matrices of size 2) with discriminant $-|2 T|$. Let $-\Delta(T)$ be the discriminant of the quadratic field $K=\boldsymbol{Q}(\sqrt{-|2 T|})$. Put $|2 T|=\Delta(T) \mathfrak{f}^{2}$ with a positive integer $\mathfrak{f}$.

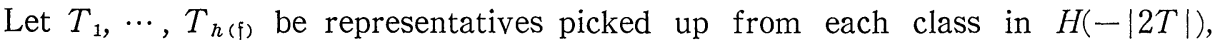
where $h(\mathfrak{f})$ denotes the cardinality of $H(-|2 T|)$. We denote by $\mathcal{O}_{K}(\mathfrak{f})$ the order of $K$ with discriminant $-\Delta(T) \mathfrak{f}^{2}$, and by $w(\mathfrak{f})$ the number of roots of unity contained in $\mathcal{O}_{K}(\mathfrak{f})$. For $\mathfrak{f}=1$, we write $h(1)=h_{K}$ and $w(1)=w_{K}$. Let $\mathscr{M}_{K}(\mathfrak{f})$ be the set of all full modules in $K$ with coefficient ring $\mathcal{O}_{K}(\mathfrak{f})$. Here, a full module means a $\boldsymbol{Z}$-module of rank 2 , and for a full module $M$ contained in $K$, its coefficient ring is defined by

$$
\mathcal{O}_{M}=\{\alpha \in K \mid \alpha M \subset M\} .
$$

As is well known (see, for example, Borevich-Shafarevich [2]), $\mathscr{M}_{K}(\mathfrak{f})$ modulo scalar multiplication by $K^{\times}$forms a finite abelian group $\mathcal{C}_{K}(\mathfrak{f})$, which is isomorphic to $H(-|2 T|)$.

Let $\phi: H(-|2 T|) \rightarrow C^{\times}$be an arbitrary character. By (2.1), (2.4), (2.5) and (2.18), we have :

$$
\begin{aligned}
& \sum_{j=1}^{h(\mathfrak{f})} \psi\left(T_{j}\right) a\left(T_{j},[f]\right)=(w(\mathfrak{f}) / 2) \xi|T|^{k-(3 / 2)} \prod_{p} A(p) \\
& \quad \cdot \sum_{\nu=1}^{\infty} \sum_{\substack{d^{2} \mid \nu \\
d>0}}\left\{C(\phi, \nu) a\left(\nu d^{-2}\right) \nu^{1-k} \prod_{p \mid \nu} A(p)^{-1} B(p) \prod_{p \backslash d} B(p)^{-1} S_{p}\left(\nu,[d]_{p}\right)\right\} .
\end{aligned}
$$

Here $\xi$ is the constant (2.3), $A(p)$ (resp. $B(p)$ ) denotes the right hand side of (2.20) (resp. (2.21)) with $\gamma=0$, and

$$
C(\phi, \nu)=2 w(\mathfrak{f})^{-1} \sum_{j=1}^{h(\mathfrak{f})} \phi\left(T_{j}\right)^{\#}\left\{(r, s) \in \mathscr{Q} \mid T\left[\begin{array}{l}
r \\
s
\end{array}\right]=\nu\right\} .
$$


Note that

$$
\prod_{p} S_{p}(\nu, 1)=\prod_{p} A(p) \prod_{p \mid \nu} A(p)^{-1} B(p)
$$

Lemma 3. $C(\phi, \nu)$ is multiplicative with respect to $\nu$.

Proof. By (2.24), we have

$$
C(\psi, \nu)=\sum_{a \in \mathscr{R}(\nu)} \phi((\nu, a, n(a))),
$$

where

$$
\mathscr{R}(\nu)=\left\{\begin{array}{ll}
a \bmod 2 \nu \mid(*) & \begin{array}{l}
a^{2} \equiv-|2 T|(\bmod 4 \nu), \\
\text { g.c.d. }\left(\nu, a,\left(a^{2}+|2 T|\right) / 4 \nu\right)=1
\end{array}
\end{array}\right\},
$$

and $(\nu, a, n(a))$ denotes the quadratic form $\nu x^{2}+a x y+n(a) y^{2}$; for each $a$, we put $n(a)=\left(a^{2}+|2 T|\right) / 4 \nu$ (cf. Landau [7]). It is easily verified that the property (*) depends only on the residue of $a$ modulo $2 \nu$. Moreover, for relatively prime positive integers $\nu_{1}, \nu_{2}$, the mapping:

$$
\begin{aligned}
& \mathscr{R}\left(\nu_{1} \nu_{2}\right) \longrightarrow \mathscr{R}\left(\nu_{1}\right) \times \mathscr{R}\left(\nu_{2}\right) \\
& a \bmod 2 \nu_{1} \nu_{2} \longmapsto\left(a \bmod 2 \nu_{1}, a \bmod 2 \nu_{2}\right)
\end{aligned}
$$

is bijective. Therefore it is sufficient to prove the following: if $a \longleftrightarrow\left(a_{1}, a_{2}\right)$ under (2.26), then

$$
\left(\nu_{1}, a_{1}, n\left(a_{1}\right)\right) \circ\left(\nu_{2}, a_{2}, n\left(a_{2}\right)\right)=\left(\nu_{1} \nu_{2}, a, n(a)\right),
$$

where $\circ$ denotes the product in $H(-|2 T|)$.

For $c_{1}, \cdots, c_{m} \in \boldsymbol{C}$, we put $\left\{c_{1}, \cdots, c_{m}\right\}=\boldsymbol{Z} c_{1}+\cdots+\boldsymbol{Z}_{c_{m}}$ (a $\boldsymbol{Z}$-module). Then, putting $-\Delta(T)=D$,

$$
\begin{aligned}
& \left\{\nu_{1},\left(a_{1}-\mathfrak{f} \sqrt{D}\right) / 2\right\}\left\{\nu_{2},\left(a_{2}-\mathfrak{f} \sqrt{D}\right) / 2\right\} \\
= & \left\{\nu_{1},(a-\mathfrak{f} \sqrt{D}) / 2\right\}\left\{\nu_{2},(a-\mathfrak{f} \sqrt{D}) / 2\right\} \quad\left(\because a \equiv a_{j}\left(\bmod 2 \nu_{j}\right)\right) \\
= & \left\{\nu_{1} \nu_{2}, \nu_{1}(a-\mathfrak{f} \sqrt{D}) / 2, \nu_{2}(a-\mathfrak{f} \sqrt{D}) / 2,\left(a^{2}+\mathfrak{f}^{2} D-2 a \mathfrak{f} \sqrt{D}\right) / 4\right\} \\
= & \left\{\nu_{1} \nu_{2},(a-\mathfrak{f} \sqrt{D}) / 2,\left(a^{2}+\mathfrak{f}^{2} D-2 a \mathfrak{f} \sqrt{D}\right) / 4\right\} \quad\left(\because\left(\nu_{1}, \nu_{2}\right)=1\right) \\
= & \left\{\nu_{1} \nu_{2},(a-\mathfrak{f} \sqrt{D}) / 2,\left(\mathfrak{f}^{2} D-a^{2}\right) / 4\right\} .
\end{aligned}
$$

Since $a^{2} \equiv \mathfrak{f}^{2} D\left(\bmod 4 \nu_{1} \nu_{2}\right)$, the above module is equal to $\left\{\nu_{1} \nu_{2},(a-\mathfrak{f} \sqrt{D}) / 2\right\}$. This implies (2.27) through the isomorphism $\mathcal{C}_{K}(\mathfrak{f}) \cong H(-|2 T|)$.

Since $f$ is an eigen cusp form, (2.23) with Lemma 3 yields the following product expression:

$$
\sum_{j=1}^{h(\mathfrak{f})} \phi\left(T_{j}\right) a\left(T_{j},[f]\right)=(w(\mathfrak{f}) / 2) \xi|T|^{k-(3 / 2)} \prod_{p} A(p) G(p),
$$

where 


$$
\begin{gathered}
G(p)=\sum_{\delta=0}^{\infty} \sum_{\substack{d^{2} 2_{p} p^{\delta} \\
d>0}}\left\{C\left(\phi, p^{\delta}\right) a\left(p^{\delta} d^{-2}\right) p^{\delta(1-k)} \cdot\left(\prod_{p \backslash p^{\delta}} A(p)^{-1} B(p)\right)\right. \\
\left.\cdot\left(\prod_{p \backslash d} B(p)^{-1} S_{p}\left(p^{\delta}, d\right)\right)\right\},
\end{gathered}
$$

and $A(p), B(p)$ are the same as in (2.23).

First we compute $G(p)$ for $p \nmid \mathfrak{f}$. If $p \nmid \mathfrak{f}$, then for each $\delta \geqq 1$ we have the following: if $\chi(p)=1$ and $(p)=\mathfrak{p} \overline{\mathfrak{p}}$ in $\mathcal{O}_{K}(\mathfrak{f})$, then $C\left(\psi, p^{\delta}\right)=\psi(\mathfrak{p})^{\delta}+\psi(\overline{\mathfrak{p}})^{\delta}$; if $\chi(p)=0$ and $(p)=\mathfrak{p}^{2}$ in $\mathcal{O}_{K}(\mathfrak{f})$, then $C(\phi, p)=\phi(\mathfrak{p})$ and $C\left(\phi, p^{\delta}\right)=0$ for $\delta \geqq 2$; if $\chi(p)=-1$, then $C\left(\phi, p^{\delta}\right)=0$ for $\delta \geqq 1$. (Here, $\mathfrak{p}$ denotes a prime ideal in $\mathcal{O}_{K}(\mathfrak{f})$ lying above $p$.) The proof is similar to that of Part I, Lemma 5(2), with $\nu$ running over all positive integers relatively prime to $f$. We note that the uniqueness of the prime decomposition holds in $\mathcal{O}_{K}(\mathfrak{f})$ for each $p \nmid \mathfrak{f}$. (Cf. Proposition in $\S 3$ below where another proof is given.) Hence

$$
G(p)=1 \quad \text { if } \quad p \times \mathfrak{f}, \chi(p)=-1 .
$$

Suppose $p \nmid \mp, \chi(p)=0$. Then

$$
G(p)=1+\psi(p) a(p) p^{1-k} A(p)^{-1} B(p) .
$$

In this case we have $A(p)=1-p^{2-2 k}, B(p)=1-p^{1-k}$, so

$$
G(p)=\left(1+p^{1-k}\right)\left(1+a(p) \phi(\mathfrak{p}) p^{1-k}+\phi(\mathfrak{p})^{2} p^{1-k}\right) .
$$

Next suppose $p \nmid \mathfrak{f}, \chi(p)=1$. Then $A(p)=1+p^{1-k}, B(p)=1$ and

$$
G(p)=1+\sum_{\delta=1}^{\infty}\left[\left\{\psi(\mathfrak{p})^{\delta}+\psi(\bar{p})^{\delta}\right\} p^{\delta(1-k)}\left(1+p^{1-k}\right)^{-1} \sum_{\substack{d^{2} \backslash p \\ d>0}}\left\{a\left(p^{\delta} d^{-2}\right) \prod_{p \nmid d} S_{p}\left(p^{\delta}, d\right)\right\}\right] .
$$

Since $p \nmid|2 T|$, we have $S_{p}\left(p^{\delta}, d\right)=\mu(d)$ if $p \mid d$, by (2.19). Thus, if $p \nmid \mathfrak{f}, \chi(p)=1$, then

$$
\begin{aligned}
G(p)=1+\left(1+p^{1-k}\right)^{-1}[ & \{\phi(\mathfrak{p})+\psi(\overline{\mathfrak{p}})\} p^{1-k} a(p) \\
& \left.+\sum_{\delta=2}^{\infty}\left\{\phi(\mathfrak{p})^{\delta}+\psi(\overline{\mathfrak{p}})^{\delta}\right\} p^{\delta(1-k)}\left(a\left(p^{\delta}\right)-a\left(p^{\delta-2}\right)\right)\right] .
\end{aligned}
$$

The above summation is equal to $F_{p}$ for $\chi(p)=1$ defined in Part I, (3.21). Namely,

We put

$$
\begin{aligned}
G(p)= & \left(1+p^{1-k}\right)^{-1}\left(1-a(p) \psi(\mathfrak{p}) p^{1-k}+\phi(\mathfrak{p})^{2} p^{1-k}\right)^{-1} \\
& \cdot\left(1-a(p) \phi(\overline{\mathfrak{p}}) p^{1-k}+\phi(\overline{\mathfrak{p}})^{2} p^{1-k}\right)^{-1} \\
& \cdot\left(1-p^{1-k}\right)\left(1-a(p) p^{1-k}+p^{1-k}\right)\left(1+a(p) p^{1-k}+p^{1-k}\right) .
\end{aligned}
$$

$$
g_{\psi}=w(\mathfrak{f})^{-1} \sum_{j=1}^{h(\mathfrak{f})} \psi\left(T_{j}\right) \vartheta_{T_{j}}
$$


which is a modular form of weight 1 and character $\left(\frac{-|2 T|}{*}\right)$ with respect to the congruence subgroup $\Gamma_{0}(|2 T|)$ of $\Gamma_{1}$. Let

$$
g_{\psi}(z)=\sum_{n=0}^{\infty} t(\psi, n) \mathrm{e}(n z)
$$

be its Fourier expansion. By (2.24) we have

$$
t(\psi, n)=\sum_{\substack{d^{2} \backslash n \\ d>0}} C\left(\psi, n d^{-2}\right) \quad \text { for } \quad n \geqq 1,
$$

hence $t(\psi, n)$ is also multiplicative.

As in Part I (3.22), (3.22a), we see from (2.30), (2.31), (2.33) that: if $p \nmid \mathfrak{f}$, then

$$
A(p) G(p)=\text { the } p \text {-factor of } \frac{L(k-1, \chi) D\left(k-1, f, g_{\psi}\right)}{L_{2}(2 k-2, f)} \text {. }
$$

\begin{tabular}{|c|c|c|c|c|}
\hline & $1 \leqq u \leqq \alpha-1$ & $u=\alpha$ & $u=\alpha+1$ & $u \geqq \alpha+2$ \\
\hline$\delta \geqq 1$ & $\varphi\left(p^{u}\right)\left(1-p^{1-k}\right)$ & $\begin{array}{l}-p^{\alpha-1}\left(1-p^{1-k}\right) \\
+p^{\alpha}\left[1+\left\{2^{-1} \chi(p)(1+\chi(p))-1\right\} p^{1-k}\right]\end{array}$ & \multirow{2}{*}{$-\chi(p)^{2} p^{\alpha}$} & \multirow{2}{*}{0} \\
\hline$\delta=0$ & \multicolumn{2}{|c|}{$\begin{array}{l}-p^{u-1}\left(1-p^{1-k}\right) \\
+p^{u}\left(1-p^{2-2 k}\right)\left(1-\chi(p) p^{1-k}\right)^{-1} p^{(\alpha-u)(3-2 k)} M\left(p^{\alpha-u}\right)\end{array}$} & & \\
\hline
\end{tabular}

Now we compute the remaining $p$-factors. Suppose $p^{\alpha} \| \mathfrak{f}, \alpha \geqq 1$.

LEMma 4. $S_{p}\left(p^{\delta+2 u}, p^{u}\right)(\delta \geqq 0, u \geqq 1)$ takes the following values:

Proof. The above table is a direct consequence of (2.19), (2.20), (2.21); verification is easy if $p$ is an odd prime or if $p=2$ and $\chi(2) \neq 0$. For the case $p=2$ and $\chi(2)=0$, note that $2^{2 \alpha+2} \chi l$ in (2.4). In fact, if $l=2^{2 \alpha+2} l^{\prime}$ with $l^{\prime} \in \boldsymbol{Z}$, then $m^{2}-2^{2 \alpha+4} l^{\prime} n=2^{2 \alpha+2} d$ with $d \equiv 2$ or $3(\bmod 4)$, so $m=2^{\alpha+1} m^{\prime}$ for some $m^{\prime} \in \boldsymbol{Z}$ and $\left(m^{\prime}\right)^{2}-4 l^{\prime} n=d,\left(m^{\prime}\right)^{2} \equiv d(\bmod 4)$, a contradiction.

Hereafter in this section, we fix $\phi$ and put

and

$$
\begin{aligned}
& c(n)=C(\phi, n), \quad t(n)=t(\psi, n), \\
& \mathcal{S}(u)=\sum_{\delta=0}^{\infty} a\left(p^{\delta}\right) c\left(p^{\delta+2 u}\right) p^{\delta(1-k)},
\end{aligned}
$$

i.e.,

$$
H(p)=p^{\alpha(2 k-3)}\left(1-\chi(p) p^{1-k}\right)\left(1-p^{1-k}\right)^{-1} A(p) G(p),
$$

$$
H(p)=\left(1+p^{1-k}\right) M\left(p^{\alpha}\right) G(p) .
$$


Remark 2. By Proposition in $\S 3$ below, the series $\mathcal{S}(u)$ is a rational function of $p^{-k}$ with coefficients lying in $\boldsymbol{Q}\left(a(n), \phi\left(T_{j}\right) \mid n \geqq 1,1 \leqq j \leqq h(\mathfrak{f})\right)$. But we do not use this fact here.

By (2.29), (3.39) we have:

$$
\begin{aligned}
H(p)=p^{\alpha(2 k-3)}\left(1-\chi(p) p^{1-k}\right)( & \left.1-p^{1-k}\right)^{-1}\left\{A(p)+\sum_{\delta=1}^{\infty} a\left(p^{\grave{o}}\right) c\left(p^{\grave{o}}\right) p^{\grave{\delta}(1-k)}\left(1-p^{1-k}\right)\right. \\
& \left.+\sum_{u=1}^{\alpha+1} \sum_{\delta=0}^{\infty} a\left(p^{\delta}\right) c\left(p^{\delta+2 u}\right) p^{(\hat{o}+2 u)(1-k)} S_{p}\left(p^{\delta+2 u}, p^{u}\right)\right\} .
\end{aligned}
$$

Lemma 4 shows that $S_{p}\left(p^{\delta+2 u}, p^{u}\right)=S_{p}\left(p^{1+2 u}, p^{u}\right)$ for all $\delta \geqq 1$, so

$$
\begin{aligned}
& H(p)=\left(1+p^{1-k}\right) M\left(p^{\alpha}\right)+p^{\alpha(2 k-3)}\left(1-\chi(p) p^{1-k}\right)(\mathcal{S}(0)-1) \\
& \quad \div p^{\alpha(2 k-3)}\left(1-\chi(p) p^{1-k}\right)\left(1-p^{1-k}\right)^{-1} \sum_{u=1}^{\alpha} c\left(p^{2 u}\right) p^{2 u(1-k)}\left\{S_{p}\left(p^{2 u}, p^{u}\right)-S_{p}\left(p^{2 u+1}, p^{u}\right)\right\} \\
& \quad \div p^{\alpha(2 k-3)}\left(1-\chi(p) p^{1-k}\right)\left(1-p^{1-k}\right)^{-1} \sum_{u=1}^{a+1} p^{2 u(1-k)} S_{p}\left(p^{2 u+1}, p^{u}\right) S(u) .
\end{aligned}
$$

Here we used $S_{p}\left(p^{2 u}, p^{u}\right)-S_{p}\left(p^{2 u+1}, p^{u}\right)=0$ for $u=\alpha+1$. If $\chi(p) \neq 1$, then it is easy to see that $c\left(p^{\hat{o}}\right)=0$ for all $\delta \geqq 2 \alpha+2$ (cf. Proposition in $\S 3$ below), hence $\mathcal{S}(\alpha+1)=0$. Thus we have always

$$
\chi(p)^{2}\left(1-\chi(p) p^{1-k}\right)\left(1-p^{1-k}\right)^{-1} \mathcal{S}(\alpha+1)=\mathcal{S}(\alpha+1) .
$$

By the same reason, $\mathcal{S}(\alpha)=c\left(p^{2 \alpha}\right)$ if $\chi(p) \neq 1$. So the terms in (2.40) corresponding to $u=\alpha$ have the form :

$$
\begin{aligned}
& p^{\alpha(2 k-3)}\left(1-\chi(p) p^{1-k}\right)\left(1-p^{1-k}\right)^{-1} c\left(p^{2 \alpha}\right) p^{2 \alpha(1-k)}\left\{S_{p}\left(p^{2 \alpha}, p^{\alpha}\right)-S_{p}\left(p^{2 \alpha+1}, p^{\alpha}\right)\right\} \\
& \quad+p^{\alpha(2 k-3)}\left(1-\chi(p) p^{1-k}\right)\left(1-p^{1-k}\right)^{-1} p^{2 \alpha(1-k)} S_{p}\left(p^{2 \alpha+1}, p^{\alpha}\right) S(\alpha) \\
& =p^{1-k} c\left(p^{2 \alpha}\right)+\left(1-p^{-1}+\chi(p) p^{-k}\right) S(\alpha) .
\end{aligned}
$$

For $1 \leqq u \leqq \alpha-1$, by Lemma 4 we have

$$
\begin{aligned}
& p^{a(2 k-3)} p^{2 u(1-k)}\left(1-\chi(p) p^{1-k}\right)\left(1-p^{1-k}\right)^{-1}\left\{S_{p}\left(p^{2 u}, p^{u}\right)-S_{p}\left(p^{2 u+1}, p^{u}\right)\right\} \\
& \quad=\left(1+p^{1-k}\right) M\left(p^{\alpha-u}\right)-p^{(\alpha-u)(2 k-3)}\left(1-\chi(p) p^{1-k}\right) \\
& \quad=p^{1-k} M\left(p^{\alpha-u}\right)+M\left(p^{\alpha-u-1}\right) .
\end{aligned}
$$

Here we used the following equality:

$$
M\left(p^{u}\right)-M\left(p^{u-1}\right)=p^{u(2 k-3)}\left(1-\chi(p) p^{1-k}\right) .
$$

Thus by (2.40) and Lemma 4 we see: 


$$
\begin{aligned}
H(p)= & p^{1-k} M\left(p^{\alpha}\right)+M\left(p^{\alpha-1}\right)+\sum_{u=1}^{\alpha-1} c\left(p^{2 u}\right)\left\{p^{1-k} M\left(p^{\alpha-u}\right)+M\left(p^{\alpha-u-1}\right)\right\} \\
& +p^{1-k} c\left(p^{2 \alpha}\right)+p^{\alpha(2 k-3)}\left(1-\chi(p) p^{1-k}\right) \mathcal{S}(0) \\
& +\sum_{u=1}^{\alpha-1} p^{(\alpha-u)(2 k-3)}\left(1-p^{-1}\right)\left(1-\chi(p) p^{1-k}\right) \mathcal{S}(u) \\
& +\left(1-p^{-1}+\chi(p) p^{-k}\right) \mathcal{S}(\alpha)-p^{2-2 k} \mathcal{S}(\alpha+1) .
\end{aligned}
$$

By (2.43), for each $u \geqq 1$ we have

$$
\begin{array}{r}
M\left(p^{u}\right)-M\left(p^{u-1}\right)+p^{2-2 k}\left(M\left(p^{u}\right)-M\left(p^{u+1}\right)\right) \\
=p^{u(2 k-3)}\left(1-p^{-1}\right)\left(1-\chi(p) p^{1-k}\right) .
\end{array}
$$

Putting $M(x)=0$ for $x \notin Z$, the left hand side of (2.45) is equal to $1-p^{-1}+\chi(p) p^{-k}$ for $u=0$, and $-p^{2-2 k}$ for $u=-1$. Comparing these values with (2.44), we obtain

$$
\begin{aligned}
H(p)= & M\left(p^{\alpha}\right)\left\{p^{1-k}+\mathcal{S}(0)-p^{2-2 k} \mathcal{S}(1)\right\}+\sum_{u=1}^{\alpha} M\left(p^{\alpha-u}\right) \\
& \cdot\left\{c\left(p^{2 u}\right) p^{1-k}+c\left(p^{2 u-2}\right)-\mathcal{S}(u-1)+\left(1+p^{2-2 k}\right) \mathcal{S}(u)-p^{2-2 k} \mathcal{S}(u+1)\right\}
\end{aligned}
$$

By (2.36), $c\left(p^{\delta}\right)=t\left(p^{\delta}\right)-t\left(p^{\delta-2}\right)$ for $\delta \geqq 2$. Moreover $t\left(p^{2 u-1}\right)=c\left(p^{2 u-1}\right)=0(1 \leqq u \leqq \alpha)$, which follows easily from the definition of $t(n)$; cf. also Proposition in $\S 3$ below. Using

$$
a\left(p^{\delta+2}\right) p^{2-2 k}+a\left(p^{\delta-2}\right)=\left(a\left(p^{2}\right) a\left(p^{\delta}\right)-p^{k-1} a\left(p^{\delta}\right)\right) p^{2-2 k} \quad(\delta \geqq 2),
$$

we get

$$
p^{1-k}+\mathcal{S}(0)-p^{2-2 k} \mathcal{S}(1)=\left(1+p^{1-k}+p^{2-2 k}-a\left(p^{2}\right) p^{2-2 k}\right) \sum_{\delta=0}^{\infty} a\left(p^{\delta}\right) t\left(p^{\delta}\right) p^{\delta(1-k)},
$$

and

$$
\begin{aligned}
& c\left(p^{2 u}\right) p^{1-k}+c\left(p^{2 u-2}\right)-\mathcal{S}(u-1)+\left(1+p^{2-2 k}\right) \mathcal{S}(u)-p^{2-2 k} \mathcal{S}(u+1) \\
& =\left(1+p^{1-k}+p^{2-2 k}-a\left(p^{2}\right) p^{2-2 k}\right) \sum_{\delta=0}^{\infty} a\left(p^{\delta}\right)\left(t\left(p^{\delta+2 u}\right)-t\left(p^{\delta+2 u-2}\right)\right) p^{\delta(1-k)}
\end{aligned}
$$

for $1 \leqq u \leqq \alpha$. Thus (2.46), with (2.39), implies

$$
\begin{aligned}
A(p) G(p)=p^{\alpha(3-2 k)}\left(1-\chi(p) p^{1-k}\right)^{-1}\left(1-p^{1-k}\right)\left(1+p^{1-k}+p^{2-2 k}-a\left(p^{2}\right) p^{2-2 k}\right) \\
\cdot\left\{M\left(p^{\alpha}\right) \sum_{\delta=0}^{\infty} a\left(p^{\delta}\right) t\left(p^{\delta}\right) p^{\delta(1-k)}\right. \\
\left.+\sum_{u=1}^{\alpha} M\left(p^{\alpha-u}\right) \sum_{\delta=0}^{\infty} a\left(p^{\delta}\right)\left(t\left(p^{\delta+2 u}\right)-t\left(p^{\delta+2 u-2}\right)\right) p^{\delta(1-k)}\right\} .
\end{aligned}
$$

Noting that

$$
L_{2}(2 k-2, f)^{-1}=\Pi_{p}\left(1-p^{1-k}\right)\left(1+p^{1-k}+p^{2-2 k}-a\left(p^{2}\right) p^{2-2 k}\right),
$$


by (2.28), (2.37), (2.47) we have:

$$
\begin{aligned}
\sum_{j=1}^{h(\mathfrak{f})} \psi\left(T_{j}\right) a\left(T_{j},[f]\right)= & (-1)^{k / 2} \frac{(k-1) !}{(2 k-2) !}(2 \pi)^{k-1} w(\mathfrak{f}) \Delta(T)^{k-(3 / 2)} \frac{L(k-1, \chi)}{L_{2}(2 k-2, f)} \\
& \cdot \sum_{s \mid \mathfrak{f}} M\left(\mathfrak{f} s^{-1}\right) \sum_{t \mid s} \mu(t) D\left(k-1, f, g_{\psi}^{(s / t)}\right),
\end{aligned}
$$

where

$$
g_{\psi}^{(v)}(z)=\sum_{n=0}^{\infty} t\left(\psi, n v^{2}\right) \mathrm{e}(n z)
$$

Thus we obtain (1.4) for a normalized eigen cusp form $f$. Observe that the right hand side of (2.48) gives an Euler-product expression.

\section{$\S 3$. Proof of Theorem 1: The Case $\Phi f \neq 0$.}

In this section we treat the case $f=G_{k}$. Since (1.4) is equivalent to (2.48), we shall prove:

LEMMA 5. Let

$$
V(\psi)=\sum_{s \mid \mathfrak{f}} M\left(\mathfrak{\mathfrak { f }} s^{-1}\right) \sum_{t \mid s} \mu(t) D\left(k-1, G_{k}, g_{\psi}^{(s / t)}\right) .
$$

(1) If $\phi=\phi_{0}$ is trivial, then:

$$
V\left(\phi_{0}\right)=M(\mathfrak{f}) \frac{\zeta_{K}(k-1) \zeta_{K}(0)}{L(k-1, \chi)} \mathfrak{f} \prod_{p / \mathfrak{f}}\left(1-\chi(p) p^{-1}\right) .
$$

(2) If $\phi$ is non-trivial, then: $V(\phi)=0$.

Here $K=\boldsymbol{Q}(\sqrt{-|2 T|})$ and $\zeta_{K}(s)$ is the Dedekind zeta function of $K$.

Suppose Lemma 5 is valid. Theorem 1 for $f=G_{k}$ is equivalent to :

$$
\begin{aligned}
& a\left(T,\left[G_{k}\right]\right)=h(\mathfrak{f})^{-1} w(\mathfrak{f})(-1)^{k / 2} \frac{(k-1) !}{(2 k-2) !}(2 \pi)^{k-1} \Delta(T)^{k-(3 / 2)} \frac{L(k-1, \chi)}{L_{2}\left(2 k-2, G_{k}\right)} \\
& \cdot \sum_{\psi} \bar{\psi}(T) V(\psi),
\end{aligned}
$$

where $\phi$ runs over all characters of $H(-|2 T|)$. By Lemma 5 which we assume now, the right hand side of (3.1) is equal to:

$$
\frac{w(\mathfrak{f})}{h(\mathfrak{f})}(-1)^{k / 2} \frac{(k-1) !}{(2 k-2) !}(2 \pi)^{k-1} \Delta(T)^{k-(3 / 2)} M(\mathfrak{f}) \frac{\zeta_{K}(k-1) \zeta_{K}(0)}{L_{2}\left(2 k-2, G_{k}\right)} \mathfrak{f} \prod_{p \backslash \mathfrak{f}}\left(1-\chi(p) p^{-1}\right) .
$$

Here,

$$
\zeta_{K}(0)=-\frac{h_{K}}{w_{K}}, \quad L_{2}\left(2 k-2, G_{k}\right)=\zeta(0) \zeta(k-1) \zeta(2 k-2), \quad \text { and }
$$




$$
\frac{h(\mathfrak{f})}{w(\mathfrak{f})}=\frac{h_{K}}{w_{K}} \mathfrak{f} \prod_{p \mid \mathfrak{f}}\left(1-\chi(p) p^{-1}\right)
$$

so (3.2) is equal to

$$
(-1)^{k / 2} \frac{(k-1) !}{(2 k-2) !} 2(2 \pi)^{k-1} \Delta(T)^{k-(3 / 2)} \frac{L(k-1, \chi)}{\zeta(2 k-2)} M(\mathfrak{f})
$$

which is exactly the formula of MaaB $[11]$ for $a\left(T,\left[G_{k}\right]\right)$. Thus Theorem 1 for $\Phi f \neq 0$ follows from Lemma 5 .

The rest of $\S 3$ is devoted to the proof of Lemma 5 . Let $T>0$ be primitive and $K=\boldsymbol{Q}(\sqrt{-|2 T|})$. Let $\Delta(T), \mathfrak{f}, H(-|2 T|), \mathcal{O}_{K}(\mathfrak{f}), h(\mathfrak{f}), w(\mathfrak{f}), h_{K}$ and $w_{K}$ be as in $\S 2$ (see before and after $(2.22)$ ). We denote by $\mathscr{M}_{K}(\mathfrak{f})$ the set of all full modules in $K$ with coefficient ring $\mathcal{O}_{K}(\mathfrak{f})$. For an order $\mathcal{O}$ in $K$ and a positive integer $\nu$, we put

and

$$
\mathscr{M}_{K}(\mathfrak{f}, \mathcal{O})=\left\{M \in \mathscr{M}_{K}(\mathfrak{f}) \mid M \subset \mathcal{O}\right\}
$$

$$
\mathscr{M}_{K}(\mathfrak{f}, \mathcal{O} ; \nu)=\left\{M \in \mathscr{M}_{K}(\mathfrak{f}, \mathcal{O}) \mid \mathrm{N}(M)=\nu\right\} \text {. }
$$

Here $\mathrm{N}(M)$ is the norm of $M$, i.e., $M \bar{M}=\mathrm{N}(M) \mathcal{O}_{M}$ (cf. (2.22)). As in $\S 2$, we put

$$
\mathcal{C}_{K}(\mathfrak{f})=\mathscr{M}_{K}(\mathfrak{f}) \text { modulo scalar multiplication by } K^{\times} \text {. }
$$

If $\mathfrak{f}^{\prime} \mid \mathfrak{f}$, the group homomorphism $\mathcal{C}_{K}(\mathfrak{f}) \rightarrow \mathcal{C}_{K}\left(\mathfrak{f}^{\prime}\right)$ induced by $M \mapsto M \mathcal{O}_{K}\left(\mathfrak{f}^{\prime}\right)$ is denoted by $\nu\left(\mathfrak{f}, \mathfrak{f}^{\prime}\right)$. It is well-known that $\nu\left(\mathfrak{f}, \mathfrak{f}^{\prime}\right)$ is surjective. Through $\mathcal{C}_{K}(\mathfrak{f}) \cong H\left(-\Delta(T) \mathfrak{f}^{2}\right)$, we see also $\nu\left(\mathfrak{f}, \mathfrak{f}^{\prime}\right)$ to be

$$
\nu\left(\mathfrak{f}, \mathfrak{f}^{\prime}\right): H\left(-\Delta(T) \mathfrak{f}^{2}\right) \longrightarrow H\left(-\Delta(T)\left(\mathfrak{f}^{\prime}\right)^{2}\right)
$$

To prove Lemma 5 , we investigate the values of $t(\psi, n)$, which is defined by (2.35) for an arbitrary character $\psi: H(-|2 T|) \rightarrow C^{\times}$. We need an explicit description of the elements of $\mathscr{M}_{K}(\mathfrak{f}, \mathcal{O}(\mathfrak{f}) ; n)$.

Proposition. The notation being as above, let $p$ be a prime number and $\mathfrak{g}=p^{-\alpha} \mathfrak{f}$ where $p^{\alpha} \| \mathfrak{f}$.

$$
t(\phi, n)=\sum_{M \in \mathscr{M}_{K}\left(\mathfrak{f}, \mathcal{O}_{K}(\mathfrak{f}) ; n\right)} \phi(M) \quad \text { for } n \geqq 1
$$

and $t(\psi, n)$ is multiplicative with respect to $n \geqq 1$.

(2) Let $(p)=\mathfrak{p} \bar{p}$ (resp. $\left.(p)=\mathfrak{p}^{2} ;(p)=\mathfrak{p}\right)$ be the prime decomposition of $p$ in $\mathcal{O}_{K}(\mathfrak{g})$ if $\chi(p)=1$ (resp. $\chi(p)=0 ; \chi(p)=-1$ ). Then the following assertions hold.

(i) There exists $P \in \mathscr{M}_{K}\left(\mathfrak{f}, \mathcal{O}_{K}(\mathfrak{g})\right)$ such that $P \mathcal{O}_{K}(\mathrm{~g})=\mathfrak{p}$. Moreover $P$ can be taken so that $P=\bar{P}$ if $\chi(p) \neq 1$.

(ii) Let

$$
\left\{M \in \mathscr{M}_{K}(\mathfrak{f}) \mid M \mathcal{O}_{K}(\mathrm{~g})=\mathcal{O}_{K}(\mathrm{~g})\right\}=\left\{M_{j} \mid j=1, \cdots, \kappa\right\},
$$

which is in fact a finite set and $\kappa=\kappa\left(p^{\alpha}\right)=p^{\alpha} \prod_{p \mid p^{\alpha}}\left(1-\chi(p) p^{-1}\right)$. Then, for $\delta \geqq 2 \alpha$, 
the elements of $\mathscr{M}_{K}\left(\mathfrak{f}, \mathcal{O}_{K}(\mathfrak{f}) ; p^{\delta}\right)$ are of the following form:

$$
\left.\begin{array}{ll}
\left\{p^{\alpha} M_{j} P^{\delta-2 \alpha-t} \bar{P}^{t} \mid 1 \leqq j \leqq \kappa, 0 \leqq t \leqq \delta-2 \alpha\right\} & \text { if } \chi(p)=1 ; \\
\left\{p^{\alpha} M_{j} P^{\delta-2 \alpha} \mid 1 \leqq j \leqq \kappa\right\} & \text { if } \chi(p)=0 ; \\
\left\{p^{\alpha} M_{j} P^{(\delta / 2)-\alpha} \mid 1 \leqq j \leqq \kappa\right\} & \text { for } \delta \text { even } \\
\varnothing & \text { for } \delta \text { odd }
\end{array}\right\} \text { if } \chi(p)=-1 .
$$

(3) Suppose $0 \leqq \delta \leqq 2 \alpha$. Then:

$$
\mathscr{M}_{K}\left(\mathfrak{f}, \mathcal{O}_{K}(\mathfrak{f}) ; p^{\delta}\right)=\left\{p^{\delta / 2} M \mid M \in \mathscr{M}_{K}(\mathfrak{f}) \text { such that } M \mathcal{O}_{K}\left(\mathfrak{f} p^{-\delta / 2}\right)=\mathcal{O}_{K}\left(\mathfrak{f} p^{-\delta / 2}\right)\right\}
$$

for $\delta$ even;

$$
\mathscr{M}_{K}\left(\mathfrak{f}, \mathcal{O}_{K}(\mathfrak{f}) ; p^{\delta}\right)=\varnothing \quad \text { for } \delta \text { odd }
$$

Moreover, for $0 \leqq \delta \leqq 2 \alpha, \delta$ even, we have a bijection:

$$
\begin{aligned}
\mathscr{M}_{K}\left(\mathfrak{f}, \mathcal{O}_{K}(\mathfrak{f}) ; p^{\delta}\right) & \longleftrightarrow\left(\mathcal{O}_{K}\left(\mathfrak{f} p^{-\delta / 2}\right)^{\times} / \mathcal{O}_{K}(\mathfrak{f})^{\times}\right) \times \operatorname{Ker}\left(\nu\left(\mathfrak{F}, \mathfrak{f} p^{-\delta / 2}\right)\right) \\
p^{\delta / 2} \zeta M & \longleftrightarrow\left(\zeta \mathcal{O}_{K}(\mathfrak{f})^{\times}, \text {the class containing } M\right) .
\end{aligned}
$$

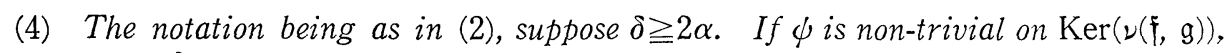
then $t\left(\phi, p^{\delta}\right)=0$; if $\phi$ is trivial on $\operatorname{Ker}(\nu(\mathfrak{f}, \mathfrak{g}))$, then

$$
t\left(\phi, p^{\delta}\right)=\left\{\begin{array}{cr}
\kappa\left(p^{\alpha}\right) \sum_{t=0}^{\delta-2 \alpha} \psi(P)^{\delta-2 \alpha-t} \psi(\bar{P})^{t} & \text { for } \chi(p)=1, \\
\kappa\left(p^{\alpha}\right) \psi(P)^{\delta} & \text { for } \chi(p)=0, \\
\kappa\left(p^{\alpha}\right) \psi(P)^{\delta / 2} & \text { for } \delta \text { even, } \chi(p)=-1, \\
0 & \text { for } \delta \text { odd }, \chi(p)=-1 .
\end{array}\right.
$$

(5) Suppose $0 \leqq \delta \leqq 2 \alpha$. If $\phi$ is trivial on $\operatorname{Ker}\left(\nu\left(\mathfrak{f}, \mathfrak{f} p^{-\delta / 2}\right)\right)$ and $\delta$ is even, then

otherwise, $t\left(\phi, p^{\delta}\right)=0$.

$$
t\left(\psi, p^{\delta}\right)=\left\{\begin{array}{lll}
p^{\delta / 2} & \text { for } & \delta<2 \alpha \\
\kappa\left(p^{\alpha}\right) & \text { for } & \delta=2 \alpha
\end{array} ;\right.
$$

Proof. (1) In (2.34), each $T_{j}$ represents the norm of each module in $\mathscr{M}_{K}\left(\mathfrak{f}, \mathcal{O}_{K}(\mathfrak{f})\right) \cap C_{j}$ exactly $w(\mathfrak{f})$ times; here $C_{\jmath} \in \mathcal{C}_{K}(\mathfrak{f})$ is the class of modules which corresponds to $T_{j}$. Hence we have (3.8). The multiplicativity of $t(\psi, n)$ follows, as we have noted, from Lemma 3 and (2.36).

(2) By the surjectivity of $\nu(\mathfrak{f}, \mathfrak{g})$, there exist $N \in \mathscr{M}_{K}(\mathfrak{f})$ and $\beta \in K^{\times}$such that $\beta N \mathcal{O}_{K}(\mathfrak{g})=\mathfrak{p}$. Since $\mathfrak{p} \subset \mathcal{O}_{K}(\mathfrak{g})$, we have $\beta N \subset \mathcal{O}_{K}(\mathfrak{g})$. Thus $P=\beta N$ is the desired one. If $\chi(p) \neq 1$, then $\bar{p}=\mathfrak{p}$. Replacing $P$ by $P+\bar{P}$ if necessary, we may assume $P=\bar{P}$ in this case. This proves (i).

We prove (ii) in the case $\chi(p)=1$; other cases are similar. For the finiteness 
of the set (3.9), see, for example, Borevich-Shafarevich [2] (Problems 8, 9 of Sec. 7, Chap. 2). The coefficient ring of $p^{\alpha} M_{j} P^{\delta-2 \alpha-t} \bar{P}^{t}$ is $\mathcal{O}_{K}(\mathfrak{f})$ since $M_{j}$ and $P$ lie in $\mathscr{M}_{K}(\mathfrak{f})$. Observe that the mapping $\mathscr{M}_{K}(\mathfrak{f}) \rightarrow \mathscr{M}_{K}(\mathrm{~g})\left(N \mapsto N \mathcal{O}_{K}(\mathrm{~g})\right)$ preserves the norm. Now the module

$$
\left(p^{\alpha} M_{\jmath} P^{\delta-2 \alpha-t} \bar{P}^{t}\right) \mathcal{O}_{K}(\mathfrak{g})=\mathfrak{p}^{\alpha+\delta-2 \alpha-t} \overline{\mathfrak{p}}^{\alpha+t}
$$

has norm $p^{\delta}$, so the norm of $p^{\alpha} M_{j} P^{\delta-2 \alpha-t} \bar{P}^{t}$ is equal to $p^{\delta}$. Conversely, take a module $N^{\prime} \in \mathscr{M}_{K}\left(\mathfrak{f}, \mathcal{O}_{K}(\mathfrak{f}) ; p^{\delta}\right)$. Then :

there exists some $N \in \mathscr{M}_{K}\left(\mathfrak{f}, \mathcal{O}_{K}(\mathfrak{g})\right)$ such that $N^{\prime}=p^{\alpha} N$.

Assuming (*), we proceed as follows. Noting that $N$ has norm $p^{\delta-2 \alpha}$, we have

$$
N \mathcal{O}_{K}(\mathrm{~g})=\mathfrak{p}^{\delta-2 \alpha-t} \overline{\mathrm{p}}^{t} \text { for some } t, \quad 0 \leqq t \leqq \delta-2 \alpha .
$$

Hence $N P^{-(\delta-2 \alpha-t)} \bar{P}^{-t}$ coincides with some $M_{j}$. Thus we have

$$
N^{\prime}=p^{\alpha} N=p^{\alpha} M_{j} P^{\delta-2 \alpha-t} \bar{P}^{t} .
$$

To prove $(*)$, we consider the generators of $N^{\prime}$; we have

$$
N^{\prime}=s\{a,(-b+\mathfrak{f} \sqrt{-\Delta(T)}) / 2\},
$$

where $a, b, s$ are integers such that $p^{\delta}=a s^{2}, b^{2}-4 a c=-\Delta(T) \mathfrak{f}^{2}$ with an integer $c$, and $\{x, y\}=\boldsymbol{Z} x+\boldsymbol{Z} y$. Cf. Borevich-Shafarevich [2] (Sec. 7, Chap. 2). By $p^{\delta}=a s^{2}$, we have $p^{\alpha} \mid a s$. Furthermore $(b s)^{2}-4\left(a s^{2}\right) c=-\Delta(T)\left(\mathrm{g} p^{\alpha} s\right)^{2}$ implies $p^{\alpha} \mid b s$. Putting $a s=p^{\alpha} a^{\prime}, b s=p^{\alpha} b^{\prime}$, we have $N^{\prime}=p^{\alpha} N$ with

$$
N=\left\{a^{\prime},\left(-b^{\prime}+s g \sqrt{-\Delta(T)}\right) / 2\right\} .
$$

One verifies easily that the right hand side is contained in $\mathcal{O}_{K}(\mathrm{~g})$. By $N=p^{-\alpha} N^{\prime}$, the coefficient ring of $N$ is $\mathcal{O}_{K}(\mathfrak{f})$. Thus $(*)$ is proved.

(3) For a positive integer $\nu, \mathscr{M}_{K}\left(\mathfrak{f}, \mathcal{O}_{K}(\mathfrak{f}) ; \nu\right) \neq \varnothing$ is equivalent to the existence of integers $s>0, a>0, b, c$ satisfying the conditions:

$$
\nu=a s^{2}, \quad b^{2}-4 a c=-\Delta(T) \mathfrak{f}^{2}, \quad(a, b, c)=1, \quad-a \leqq b<a
$$

(Theorem 6 of Borevich-Shafarevich [2], Sec. 7, Chap. 2). Suppose such s, $a, b$, $c$ exist for $\nu=p^{\delta}, 0 \leqq \delta \leqq 2 \alpha, \delta$ odd. Then $\alpha \geqq 1$. By $p^{\delta}=a s^{2}$, we have $a=p^{\varepsilon}$ for some odd integer $\varepsilon(<2 \alpha)$. Hence by $b^{2}-4 a c=-\Delta(T) p^{2 \alpha} \mathrm{g}^{2}$, we have $p^{\varepsilon} \mid b^{2}$. We write $b=p^{\gamma} b^{\prime}$ with $2 \gamma>\varepsilon$ and $p \nmid b^{\prime}$. By $(a, b, c)=1$, we have $p \nmid c$. Thus $4 c=p\left(p^{2 \gamma-\varepsilon-1}\left(b^{\prime}\right)^{2}+\Delta(T) p^{2 \alpha-\varepsilon-1} \mathrm{~g}^{2}\right)$, which is impossible for $p \geqq 3$. If $p=2$, then we have $2 c=2^{2 \gamma-\varepsilon-1}\left(b^{\prime}\right)^{2}+\Delta(T) 2^{2 \alpha-\varepsilon-1} g^{2}$. The right hand side is congruent to 0,1 , or 3 modulo 4 , a contradiction. Thus $\mathscr{M}_{K}\left(\mathfrak{f}, \mathcal{O}_{K}(\mathfrak{f}) ; p^{\delta}\right)=\varnothing$ if $\delta$ is odd, $0 \leqq \delta \leqq 2 \alpha$. Next suppose that $\delta$ is even, $0 \leqq \delta \leqq 2 \alpha$. For $M \in \mathscr{M}_{K}(\mathfrak{f})$ such that $M O_{K}\left(\mathfrak{f} p^{-\delta / 2}\right)=$ $\mathcal{O}_{K}\left(\mathfrak{f} p^{-\delta / 2}\right)$, the norm of $p^{\delta / 2} M$ is $p^{\delta}$ by the same reason stated before (*) in the proof of (2). Conversely, take any $M^{\prime} \in \mathscr{M}_{K}\left(\mathfrak{f}, \mathcal{O}_{K}(\mathfrak{f}) ; p^{\delta}\right)$. Then there exists some $M \in \mathscr{M}_{K}\left(\mathfrak{f}, \mathcal{O}_{K}\left(\mathfrak{f} p^{-\delta / 2}\right)\right)$ such that $M^{\prime}=p^{\delta / 2} M$; the proof is similar to that of 
(*) in (2). Observe that $M$ has norm 1 so that $M \mathcal{O}_{K}\left(\mathfrak{f} p^{-\delta / 2}\right) \in \mathscr{M}_{K}\left(\mathfrak{f} p^{-\delta / 2}, \mathcal{O}_{K}\left(\mathfrak{f} p^{-\hat{\delta} / 2}\right) ; 1\right)$. Since $M \mathcal{O}_{K}\left(\mathfrak{f} p^{-\delta / 2}\right) \subset \mathcal{O}_{K}\left(\mathfrak{f} p^{-\delta / 2}\right)$, we have $M \mathcal{O}_{K}\left(\mathfrak{f} p^{-\delta / 2}\right)=\mathcal{O}_{K}\left(\mathfrak{f} p^{-\delta / 2}\right)$. This proves the first assertion of (3). To prove the second assertion, let $M_{j}^{\prime} \in \mathscr{M}_{K}\left(\mathfrak{f}, \mathcal{O}_{K}(\mathfrak{f}) ; p^{\delta}\right)$ $(j=1,2)$ be similar modules, i. e. $M_{1}^{\prime}=\beta M_{2}^{\prime}$ for some $\beta \in K^{\times}$; here $\delta$ is even, $0 \leqq \delta \leqq 2 \alpha$. There exist $M_{j}^{\prime \prime} \in \mathscr{M}_{K}(\mathfrak{f})$ such that $M_{j}^{\prime}=p^{\delta / 2} M_{j}^{\prime \prime}$ and $M_{j}^{\prime \prime} \mathcal{O}_{K}\left(\mathfrak{f} p^{-\delta / 2}\right)=$ $\mathcal{O}_{K}\left(\mathfrak{f} p^{-\delta / 2}\right)$, as above. Hence $M_{1}^{\prime \prime}=\beta M_{2}^{\prime \prime}$. Then multiplication by $\mathcal{O}_{K}\left(\mathfrak{f} p^{-\delta / 2}\right)$ yields

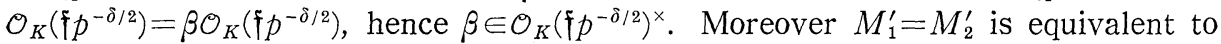
$\beta \in \mathcal{O}_{K}(\mathfrak{f}) \cap \mathcal{O}_{K}\left(\mathfrak{f} p^{-\delta / 2}\right)^{\times}=\mathcal{O}_{K}(\mathfrak{f})^{\times}$. Since each class in $\operatorname{Ker}\left(\nu\left(\mathfrak{f}, \mathfrak{f} p^{-\delta / 2}\right)\right)$ contains a module $M \in \mathscr{M}_{K}(\mathfrak{f})$ such that $M \mathcal{O}_{K}\left(\mathfrak{f} p^{-\delta / 2}\right)=\mathcal{O}_{K}\left(\mathfrak{f} p^{-\delta / 2}\right)$, the proof of (3) is completed.

Here we note the following. For $\delta=2 \alpha$, the last assertion of (3) with (2) (ii) implies that: $\left\{M_{j} \mid 1 \leqq j \leqq \kappa\right\}$ in (3.9) may be taken as $\left\{\zeta M_{j}^{\prime} \mid 1 \leqq j \leqq \kappa /(w(g) / w(\mathfrak{f}))\right.$, $\left.\zeta \mathcal{O}_{K}(\mathfrak{f})^{\times} \in \mathcal{O}_{K}(\mathfrak{g})^{\times} / \mathcal{O}_{K}(\mathfrak{f})^{\times}\right\}$such that $M_{j}^{\prime}(1 \leqq j \leqq \kappa /(w(\mathfrak{g}) / w(\mathfrak{f})))$ gives representatives of the classes in $\operatorname{Ker}(\nu(\mathfrak{f}, \mathfrak{g}))$.

By the above remark, the assertions (4), (5) follow from (1), (2), (3). Note that: if $0 \leqq \delta \leqq 2 \alpha, \delta$ even, then by the bijection in (3) we have

$$
\# \mathscr{M}_{K}\left(\mathfrak{f}, \mathcal{O}_{K}(\mathfrak{f}) ; p^{\delta}\right)=\left(w\left(\mathfrak{f} p^{-\delta / 2}\right) / w(\mathfrak{T})\right) \cdot\left(h(\mathfrak{f}) / h\left(\mathfrak{f} p^{-\delta / 2}\right)\right)= \begin{cases}p^{\delta / 2} & (\delta<2 \alpha) \\ \kappa\left(p^{\alpha}\right) & (\delta=2 \alpha)\end{cases}
$$

(cf. (3.3)).

Remark 3. In Hirzebruch-Zagier [3] (Proposition 2, Chap. 1), the assertions (4) (5) for the trivial character are obtained by a different method.

Let the notation be as above. We put

$$
L(s, \phi)=\sum_{n=1}^{\infty} t(\phi, n) n^{-s} .
$$

By Proposition (1), $L(s, \phi)$ and $D\left(s, G_{k}, g_{\psi}^{(v)}\right)$ have Euler-product expressions for $\operatorname{Re}(s)$ sufficiently large:

where

$$
L(s, \phi)=\prod_{p} L_{p}(s, \phi)
$$

$$
\begin{gathered}
L_{p}(s, \phi)=\sum_{\delta=0}^{\infty} t\left(\phi, p^{\delta}\right) p^{-\delta s} ; \\
D\left(s, G_{k}, g_{\psi}^{(v)}\right)=\prod_{p} D_{p}\left(s, G_{k}, g_{\psi}^{\left(p^{u}\right)}\right)
\end{gathered}
$$

where

$$
D_{p}\left(s, G_{k}, g_{\psi}^{\left(p^{u}\right)}\right)=\sum_{\delta=0}^{\infty} \sigma_{k-1}\left(p^{\delta}\right) t\left(\phi, p^{\delta+2 u}\right) p^{-\delta s}
$$

with the integer $u$ such that $p^{u} \| v$.

Now we prove Lemma 5 . Let $v>0$ be an integer dividing $\mathfrak{f}$, and for each prime number $p$, let $\alpha$ and $u$ be integers such that $p^{\alpha}\left\|\mathfrak{f}, p^{u}\right\| v$.

(1) Suppose first that $\phi=\phi_{0}$, the trivial character. Then, by (3.11) and Proposition (4) (5) we have (after some computation): 


$$
D\left(s, G_{k}, g_{\psi_{0}}^{(v)}\right)=\frac{\zeta_{K}(s) \zeta_{K}(s-k+1)}{L(2 s-k+1, \chi)} \prod_{p / f} R_{p}^{u}(s)
$$

where

$$
\begin{aligned}
R_{p}^{u}(s)= & \left(1-p^{k-1}\right)^{-1}\left(1-\chi(p) p^{k-1-2 s}\right)^{-1} \\
& \cdot\left[\left(1-p^{-s}\right)\left(1-p^{k-1-s}\right)\left(1-\chi(p) p^{-s}\right)\left(1-\chi(p) p^{k-1-s}\right)\right. \\
& \cdot \sum_{\delta=0}^{\alpha-u-1}\left(p^{\delta(1-2 s)}-p^{k-1+\delta(2 k-1-2 s)}\right)+\kappa\left(p^{\alpha}\right) p^{-2(\alpha-u) s} \\
& \left.\cdot\left\{\left(1-p^{k-1-s}\right)\left(1-\chi(p) p^{k-1-s}\right)-p^{(2 \alpha-2 u+1)(k-1)}\left(1-p^{-s}\right)\left(1-\chi(p) p^{-s}\right)\right\}\right] .
\end{aligned}
$$

\section{Putting}

$$
V_{p}(s, \mathfrak{f})=M\left(p^{\alpha}\right) D_{p}\left(s, G_{k}, g_{\psi_{0}}\right)+\sum_{u=1}^{\alpha} M\left(p^{\alpha-u}\right)\left(D_{p}\left(s, G_{k}, g_{\psi_{0}}^{\left(p^{u}\right)}\right)-D_{p}\left(s, G_{k}, g_{\psi_{0}}^{\left(p^{u-1}\right)}\right)\right),
$$

we see

$$
V\left(\phi_{0}\right)=\prod_{p} V_{p}(k-1, \mathfrak{f}) .
$$

By (3.12) we have

$$
\prod_{p} V_{p}(s, \mathfrak{f})=\frac{\zeta_{K}(s) \zeta_{K}(s-k+1)}{L(2 s-k+1, \chi)} \prod_{p \mid \mathfrak{f}}\left\{M\left(p^{\alpha}\right) R_{p}^{0}(s)+\sum_{u=1}^{\alpha} M\left(p^{\alpha-u}\right)\left(R_{p}^{u}(s)-R_{p}^{u-1}(s)\right)\right\} .
$$

The right hand side is a meromorphic function on the whole $s$-plane and holomorphic at $s=k-1$. By (3.13),

$$
R_{p}^{u}(k-1)=\kappa\left(p^{\alpha}\right)=p^{\alpha} \prod_{p \backslash p^{\alpha}}\left(1-\chi(p) p^{-1}\right) \quad \text { for } \quad 0 \leqq u \leqq \alpha .
$$

Hence, by (3.14) and (3.15),

$$
V\left(\phi_{0}\right)=\frac{\zeta_{K}(k-1) \zeta_{K}(0)}{L(k-1, \chi)} \prod_{p \nmid \uparrow} M\left(p^{\alpha}\right) \kappa\left(p^{\alpha}\right),
$$

which proves Lemma 5 (1).

(2) Next suppose that $\phi$ is non-trivial. In this case, it is sufficient to prove:

$$
D\left(k-1, G_{k}, g_{\psi}^{(v)}\right)=0 \quad \text { for any } v \mid \mathfrak{f} \text {. }
$$

For each $j$, the Epstein zeta function $\sum_{(m, n) \in Z^{2}}\left(T_{j}\left[\begin{array}{c}m \\ n\end{array}\right]\right)^{-s}$ is meromorphically continued to the whole $s$-plane. In particular, it is holomorphic at $s=0$, and the value at $s=0$ does not depend on $j$. Hence $L(s, \psi)$ is holomorphic at $s=0$ and $L(0, \phi)=0$. Therefore, (3.16) follows from the following

LEMMA 6. $D\left(s, G_{k}, g_{\psi}^{(v)}\right) / L(s-k+1, \phi)$ is holomorphic at $s=k-1$ for any $v / f$. (Here each function is considered as a meromorphic function on the whole 
s-plane by the analytic continuation.)

Proof. We have

$$
D\left(s, G_{k}, g_{\psi}^{(v)}\right) / L(s-k+1, \psi)=\prod_{p}\left[D_{p}\left(s, G_{k}, g_{\psi}^{\left(p^{u}\right)}\right) / L_{p}(s-k+1, \psi)\right]
$$

and

$$
\prod_{p \nmid \uparrow}\left[D_{p}\left(s, G_{k}, g_{\psi^{(p)}}^{\left(p^{u}\right)}\right) / L_{p}(s-k+1, \phi)\right]=\prod_{p \nmid \mathfrak{f}}\left[\left(1-\chi(p) p^{k-1-2 s}\right) L_{p}(s, \psi)\right]
$$

by Proposition (4) for $p \nmid \mathfrak{f}$. The right hand side of (3.18) converges absolutely and uniformly for $\operatorname{Re}(s)>k / 2$, hence holomorphic at $s=k-1$. Next we prove:

$$
\prod_{p \mid f}\left[D_{p}\left(s, G_{k}, g_{\psi}^{\left(p^{u}\right)}\right) / L_{p}(s-k+1, \psi)\right] \text { is holomorphic at } s=k-1 .
$$

This, together with what we have mentioned, implies Lemma 6. Observe that

$$
\begin{gathered}
D_{p}\left(s, G_{k}, g_{\psi}^{\left(p^{u}\right)}\right)=\left(1-p^{1-k}\right)^{-1}\left[p^{2(s-k+1) u}\left\{L_{p}(s-k+1, \phi)-\sum_{\delta=0}^{2 u-1} t\left(\psi, p^{\delta}\right) p^{-\delta(s-k+1)}\right\}\right. \\
\left.-p^{1-k+2 u s}\left\{L_{p}(s, \phi)-\sum_{\delta=0}^{2 u-1} t\left(\phi, p^{\delta}\right) p^{-\delta s}\right\}\right] .
\end{gathered}
$$

Hence, to prove (3.19), it is sufficient to see:

$$
L_{p}(s, \phi)^{-1} \text { is holomorphic at } s=0 \text { for each } p \mid \mathfrak{f} .
$$

Suppose first that $\phi$ is non-trivial on $\operatorname{Ker}\left(\nu\left(\mathfrak{f}, \mathfrak{f} p^{-\alpha}\right)\right)$. Then, by Proposition (4) (5), $L_{p}(s, \psi)$ is a polynomial of $p^{-s}$ and $L_{p}(0, \psi)>0$. Hence $(3.20)$ holds. Next suppose that $\phi$ is trivial on $\operatorname{Ker}\left(\nu\left(\mathfrak{f}, \mathfrak{f} p^{-\alpha}\right)\right)$. We prove $(3.20)$ in the case $\chi(p)=1$. (Other cases are similar.) By Proposition (4) (5), we have

$$
L_{p}(s, \phi)=\sum_{\delta=0}^{\alpha-1} p^{\delta(1-2 s)}+\kappa\left(p^{\alpha}\right) p^{-2 \alpha s}\left(1-\psi(P) p^{-s}\right)^{-1}\left(1-\phi(\bar{P}) p^{-s}\right)^{-1} .
$$

Thus, if $\phi(P) \neq 1$, then $L_{p}(s, \psi)$ is holomorphic at $s=0$ and $L_{p}(0, \phi)>0$, hence (3.20). If $\phi(P)=1$, then

where

$$
L_{p}(s, \phi)=\left(1-p^{-s}\right)^{-2} H(s) \text {, }
$$

$$
H(s)=\left(1-p^{-s}\right)^{2} \sum_{\delta=0}^{\alpha-1} p^{\delta(1-2 s)}+\kappa\left(p^{\alpha}\right) p^{-2 \alpha s} .
$$

Hence $H(0) \neq 0$ implies (3.20). This completes the proof of Lemma 6, (3.16), and Lemma 5 , hence of Theorem 1.

\section{$\S 4$. Proofs of Theorem 2 and Theorem 3 .}

The proof of Theorem 2 is entirely similar to that of Theorem 2 in Part I (cf. Remark 2 in Part I, §4); observe that each $\vartheta_{T}^{(v)}$ for $v \mid \mathfrak{f}$ is a modular form 
of weight 1 and character $\left(\frac{-|2 T|}{*}\right)$ with respect to the congruence subgroup $\Gamma_{0}(|2 T|)$ of $\Gamma_{1}(\mathrm{Li}[9]$, Lemma 1$)$.

Before the proof of Theorem 3, we note the following facts. If $\Phi f \neq 0$, then $a(T,[f]) \neq 0$ for all $T \geqq 0$ by the formula of Siegel [16], Maaß $[11,12]$. So we may suppose $\Phi f=0$. In this case, the product expression (2.28) which converges absolutely for $k \geqq 12$ shows that $\sum_{j=1}^{h(\mathfrak{f}) \cdot} \phi\left(T_{j}\right) a\left(T_{j},[f]\right) \neq 0$, hence $a\left(T_{j},[f]\right)$ $\neq 0$ for at least one $j$. This point was communicated by Professor Maaß, cf. $\S 1$.

Now we prove Theorem 3. We may assume that $\Phi f=0$ as we have just mentioned. If $\operatorname{rank} T<2$, then $T$ is unimodularly equivalent to $\left(\begin{array}{ll}1 & 0 \\ 0 & 0\end{array}\right)$ by the assumption, hence $a(T,[f])=a(1) \neq 0$. So we assume $T>0$. Then by the assumption $T$ is primitive. By (2.23), we have:

where

$$
a(T,[f])=\eta \sum_{\nu=1}^{\infty} \sum_{\substack{d^{2} / \nu \\ d>0}} N(T, \nu) a\left(\nu d^{-2}\right) \nu^{1-k} r(\nu, d),
$$

and

$$
\eta=2^{-1} w(\mathfrak{f}) \xi|T|^{k-(3 / 2)} \prod_{p} A(p), \quad N(T, \nu)=\left\{(r, s) \in \mathscr{Q} \mid T\left[\begin{array}{l}
r \\
s
\end{array}\right]=\nu\right\},
$$

$$
r(\nu, d)=\prod_{p \mid \nu} A(p)^{-1} \dot{B(p)} \prod_{p \backslash d} B(p)^{-1} S_{p}\left(\nu,[d]_{p}\right) .
$$

By the assumption,

$$
N(T, 1)=w(\mathfrak{f}) / 2 .
$$

By (2.25), for each $\nu \geqq 1$ we have:

$$
N(T, \nu) \leqq 2^{-1} w(\mathfrak{f})^{\#}\left\{x \bmod 2 \nu\left|x^{2} \equiv-\right| 2 T \mid(\bmod 4 \nu)\right\} \leqq w(\mathfrak{f}) \nu .
$$

Next we prove:

$$
|r(\nu, d)| \leqq \varphi(d) \prod_{p \mid \nu}\left(1-p^{2-k}\right)^{-1} .
$$

To prove this, it is sufficient to see the case where $\nu=p^{\delta}$ and $d=p^{u}(\delta \geqq 2 u)$ with a prime number $p$. It is clear that (4.4) holds for $\nu=d=1$, so we may assume $\delta>0$. Suppose first that $u=0$. Then

$$
r\left(p^{\delta}, 1\right)=A(p)^{-1} B(p) .
$$

Here $B(p)$, which is the right hand side of (2.21) with $\gamma=0$, satisfies $|B(p)| \leqq 1$. And $A(p)$, which is the right hand side of (2.20) with $\gamma=0$, may also be written

$$
A(p)=\left(1-p^{2-2 k}\right) \sum_{a=0}^{j} p^{a(3-2 k)}+\left(1-\chi(p) p^{1-k}\right)^{-1}\left(1-p^{2-2 k}\right) \chi(p)^{2} p^{(j+1)(3-2 k)} .
$$

Here $j \geqq-1$, and $j=-1$ implies $\chi(p) \neq 0$. Thus

$$
A(p) \geqq 1-p^{1-k} .
$$


Hence by (4.5) we have $\left|r\left(p^{\delta}, 1\right)\right| \leqq\left(1-p^{1-k}\right)^{-1}$, thus (4.4) is valid if $\nu=p^{\delta}, d=1$. Next suppose $u>0$. Then by (2.5),

$$
\left|\Lambda\left(p^{u}, p^{r}\right)\right| \leqq p^{\gamma} \varphi\left(p^{r}\right) \varphi\left(p^{u}\right) \quad \text { for } \quad r \geqq 0 .
$$

Hence by (2.16) we have

$$
\begin{aligned}
\left|S_{p}\left(p^{\delta}, p^{u}\right)\right| & \leqq \sum_{\gamma=0}^{\infty} p^{-k \gamma}\left|\Lambda\left(p^{u}, p^{i}\right)\right| \leqq \varphi\left(p^{u}\right)\left(1+\sum_{\gamma=1}^{\infty} p^{(2-k) r}\left(1-p^{-1}\right)\right) \\
& =\varphi\left(p^{u}\right)\left(1-p^{1-k}\right)\left(1-p^{2-k}\right)^{-1} .
\end{aligned}
$$

Combining this with (4.6), we obtain $\left|r\left(p^{\delta}, p^{u}\right)\right| \leqq \varphi\left(p^{u}\right)\left(1-p^{2-k}\right)^{-1}$. Hence (4.4) is proved.

We put

$$
S=\sum_{\nu=2}^{\infty}\left\{\nu \sum_{\substack{d^{2} \mid \nu \\ d>0}}\left|a\left(\nu d^{-2}\right)\right| \nu^{1-k} \varphi(d) \prod_{p \mid \nu}\left(1-p^{2-k}\right)^{-1}\right\} .
$$

We shall prove: $S<1 / 2$. If this inequality holds, then by (4.1), (4.2), (4.3), (4.4) we get $a(T,[f]) \neq 0$.

By (4.7),

where

$$
1+S=\prod_{p} S_{p}
$$

$$
\begin{aligned}
1<S_{p} & =\sum_{\delta=0}^{\infty}\left\{p^{\delta(2-k)} \prod_{p \nmid p^{\delta}}\left(1-p^{2-k}\right)^{-1}\left(\sum_{\substack{2 ! \\
d>0}}\left|a\left(p^{\delta} d^{-2}\right)\right| \varphi(d)\right)\right\} \\
& \leqq\left(1-p^{2-k}\right)^{-1} \sum_{\delta=0}^{\infty}\left\{p^{\delta(2-k)} \sum_{\substack{d^{2} \backslash>0 \\
d>0}} \sigma_{0}\left(p^{\delta} d^{-2}\right)\left(p^{\delta} d^{-2}\right)^{(k-1) / 2} \varphi(d)\right\} \\
& \leqq\left(1-p^{2-k}\right)^{-1} \sum_{\delta=0}^{\infty} p^{\delta(3-k) / 2} \sum_{u=0}^{[\delta / 2]}(\delta-2 u+1) \varphi\left(p^{u}\right) p^{u(1-k)} .
\end{aligned}
$$

By $\left|\varphi\left(p^{u}\right) p^{u(1-k)}\right| \leqq 1(0 \leqq u \leqq[\delta / 2])$ we have

$$
S_{p} \leqq\left(1-p^{2-k}\right)^{-1} \sum_{\delta=0}^{\infty} p^{\delta(3-k) / 2}(\delta+1)([\delta / 2]+1) .
$$

It is easy to see: $(\delta+1)([\delta / 2]+1) \leqq p^{3 \delta / 2}$ for $p \geqq 2, \delta \geqq 0$. Thus

$$
S_{p} \leqq\left(1-p^{2-k}\right)^{-1}\left(1-p^{3-(k / 2)}\right)^{-1} \text {. }
$$

Hence

$$
S=\prod_{p} S_{p}-1 \leqq \zeta(k-2) \zeta((k / 2)-3)-1 \leqq \zeta(10) \zeta(3)-1<1 / 2 .
$$

This completes the proof of Theorem 3 .

Remark 4. By the above proof and the formula of Siegel [16], Maab [11, 12], we see the following. Under the same assumption as in Theorem 3, we have $(-1)^{(r-1) k / 2} a(T,[f])>0$, where $r=\operatorname{rank} T$. 


\section{REFERENCES}

[1] ANdRianov, A.N., Euler products corresponding to Siegel modular forms of genus 2 (in Russian), Uspehi Mat. Nauk., 29, 43-110 (1974) (English translation in Russian Math. Surveys, 29 (1974), 45-116).

[2] Borevich, Z.I. And Shafarevich, I. R., Number Theory, New York, London: Academic Press 1966.

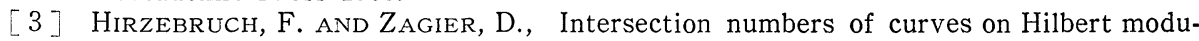
lar surfaces and modular forms of Nebentypus, Invent. Math., 36 (1976), 57-113.

[4] Klingen, H., Zum Darstellungssatz für Siegelsche Modulformen, Math. Z., 102 (1967), 30-43.

[5] Kurokawa, N., On Eisenstein series for Siegel modular groups, Proc. Japan Acad., 57A (1981), 51-55; II. Ibid., 57A (1981), 315-320.

[6] Kurokawa, N. and Mizumoto, S., On Eisenstein series of degree two, Proc. Japan Acad., 57A (1981), 134-139.

[7] Landau, E., Vorlesungen über Zahlentheorie, Bd I. Leipzig: Hirzel 1927.

[8] Langlands, R.P., On the Functional Equations Satisfied by Eisenstein Series, Lecture Notes in Mathematics, 544. Berlin/Heidelberg/New York: Springer 1976.

[9] Li, W., New forms and functional equations, Math. Ann., 212 (1975), 285-315.

[10] MaAss, H., Die Primzahlen in der Theorie der Siegelschen Modulfunktionen, Math. Ann., 124 (1951), 87-122.

[11] Masss, H., Die Fourierkoeffizienten der Eisensteinreihen zweiten Grades, Mat.-Fys. Medd. Danske Vid. Selsk., 34, nr. 7 (1964).

[12] MaAss, H., Über die Fourierkoeffizienten der Eisensteinreihen zweiten Grades, Mat.-Fys. Medd. Danske Vid. Selsk., 38, nr. 14 (1972).

[13] Mizumoto, S., Fourier coefficients of generalized Eisenstein series of degree two, I. Invent. Math., 65 (1981), 115-135.

[14] Mizumoto, S., On Eisenstein series of degree two for Hilbert-Siegel modular groups, Proc. Japan Acad., 58A (1982), 33-36.

[15] Shimura, G., The special values of the zeta functions associated with cusp forms, Comm. Pure Appl. Math., 29 (1976), 783-804.

[16] SiEgEL, C.L., Einführung in die Theorie der Modulfunktionen $n$-ten Grades, Math. Ann., 116 (1939), 617-657.

[17] Sturm, J., Special values of zeta functions and Eisenstein series of half integral weight, Amer. J. Math., 102 (1980), 219-240.

[18] ZAGIER, D., Modular forms whose Fourier coefficients involve zeta-functions of quadratic fields, Lecture Notes in Mathematics, 627, 105-169. Berlin/Heidelberg/ New York: Springer 1977.

[19] Böcherer, S., Über gewisse Siegelsche Modulformen zweiten Grades, Math. Ann., 261 (1982), 23-41.

[20] Mizumoto, S., Integrality of certain algebraic values attached to modular forms, Proc. Japan Acad., 59A (1983), 33-36.

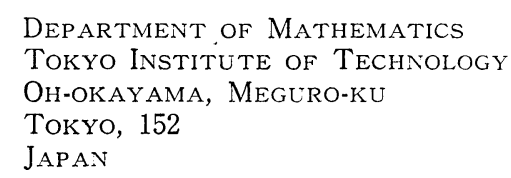

\section{Note added in proof}

Details of [20] are contained in: Mizumoto, S., On integrality of certain algebraic numbers associated with modular forms. Math. Ann., 265 (1983), 119135. 